TRANSACTIONS OF THE

AMERICAN MATHEMATICAL SOCIETY

Volume 355, Number 3, Pages 1213-1235

S 0002-9947(02)03191-4

Article electronically published on November 7, 2002

\title{
INFINITE PARTITION REGULAR MATRICES: SOLUTIONS IN CENTRAL SETS
}

\author{
NEIL HINDMAN, IMRE LEADER, AND DONA STRAUSS
}

\begin{abstract}
A finite or infinite matrix $A$ is image partition regular provided that whenever $\mathbb{N}$ is finitely colored, there must be some $\vec{x}$ with entries from $\mathbb{N}$ such that all entries of $A \vec{x}$ are in the same color class. In contrast to the finite case, infinite image partition regular matrices seem very hard to analyze: they do not enjoy the closure and consistency properties of the finite case, and it is difficult to construct new ones from old. In this paper we introduce the stronger notion of central image partition regularity, meaning that $A$ must have images in every central subset of $\mathbb{N}$. We describe some classes of centrally image partition regular matrices and investigate the extent to which they are better behaved than ordinary image partition regular matrices. It turns out that the centrally image partition regular matrices are closed under some natural operations, and this allows us to give new examples of image partition regular matrices. In particular, we are able to solve a vexing open problem by showing that whenever $\mathbb{N}$ is finitely colored, there must exist injective sequences $\left\langle x_{n}\right\rangle_{n=0}^{\infty}$ and $\left\langle z_{n}\right\rangle_{n=0}^{\infty}$ in $\mathbb{N}$ with all sums of the forms $x_{n}+x_{m}$ and $z_{n}+2 z_{m}$ with $n<m$ in the same color class. This is the first example of an image partition regular system whose regularity is not guaranteed by the Milliken-Taylor Theorem, or variants thereof.
\end{abstract}

\section{INTRODUCTION}

In 1933, R. Rado 9 characterized those (finite) matrices with rational entries that are kernel partition regular, that is, those matrices $A$ with the property that whenever $\mathbb{N}$ is finitely colored, there exists some $\vec{x}$ with monochrome entries such that $A \vec{x}=\overrightarrow{0}$. He showed that $A$ is kernel partition regular if and only if $A$ satisfies a computable property called the columns condition. (See [4] or [7] for a presentation and proof of Rado's Theorem.)

Sixty years later, several characterizations of (finite) image partition regular matrices were obtained [5]. These are the matrices $A$ with the property that whenever $\mathbb{N}$ is finitely colored, there will be some $\vec{x}$ (with entries from $\mathbb{N}$ ) such that the entries of $A \vec{x}$ are monochrome. Image partition regular matrices are of special interest because many of the classical theorems of Ramsey Theory are naturally stated as statements about image partition regular matrices. For example, Schur's Theorem [10] and the length 4 version of van der Waerden's Theorem [12] amount to the

Received by the editors May 10, 2001.

2000 Mathematics Subject Classification. Primary 05D10; Secondary 22A15, 54H13.

The first author acknowledges support received from the National Science Foundation (USA) via grant DMS-0070593. 
assertions that the matrices

$$
\left(\begin{array}{ll}
1 & 0 \\
0 & 1 \\
1 & 1
\end{array}\right) \quad \text { and } \quad\left(\begin{array}{cc}
1 & 0 \\
1 & 1 \\
1 & 2 \\
1 & 3
\end{array}\right)
$$

are image partition regular.

In 6] additional characterizations of finite image partition regular matrices were obtained. Some of these involve the notion of central sets. Central sets were introduced by Furstenberg [3] and defined in terms of notions of topological dynamics. These sets enjoy very strong combinatorial properties. (See [3. Proposition 8.21] or 7, Chapter 14].) They have a nice characterization in terms of the algebraic structure of $\beta \mathbb{N}$, the Stone-Cech compactification of $\mathbb{N}$. We shall present this characterization below, after introducing the necessary background information.

Let $(S,+)$ be an infinite discrete semigroup. We take the points of $\beta S$ to be the ultrafilters on $S$, the principal ultrafilters being identified with the points of $S$. Given a set $A \subseteq S$, we define $\bar{A}=\{p \in \beta S: A \in p\}$. The set $\{\bar{A}: A \subseteq S\}$ is a basis for the open sets (as well as a basis for the closed sets) of $\beta S$.

There is a natural extension of the operation + of $S$ to $\beta S$ making $\beta S$ a compact right topological semigroup with $S$ contained in its topological center. This says that for each $p \in \beta S$, the function $\rho_{p}: \beta S \rightarrow \beta S$ is continuous and for each $x \in S$, the function $\lambda_{x}: \beta S \rightarrow \beta S$ is continuous, where $\rho_{p}(q)=q+p$ and $\lambda_{x}(q)=x+q$. See [7] for an elementary introduction to the semigroup $\beta S$.

Any compact Hausdorff right topological semigroup $(T,+)$ has a smallest twosided ideal $K(T)$ which is the union of all of the minimal left ideals of $T$, each of which is closed [7, Theorem 2.8], and any compact right topological semigroup contains idempotents. Since the minimal left ideals are themselves compact right topological semigroups, this says in particular that there are idempotents in the smallest ideal. There is a partial ordering of the idempotents of $T$ determined by $p \leq q$ if and only if $p=p+q=q+p$. An idempotent $p$ is minimal with respect to this order if and only if $p \in K(T)$ [7, Theorem 1.59]. Such an idempotent is called simply "minimal".

Definition 1.1. Let $(S,+)$ be an infinite discrete semigroup. A set $A \subseteq S$ is central if and only if there is some minimal idempotent $p$ such that $A \in p$.

See [7] Theorem 19.27] for a proof of the equivalence of the definition above with the original dynamical definition.

We present in Theorem 1.2 a few known characterizations of finite image partition regular matrices. (There and elsewhere we take $\mathbb{N}=\{1,2,3, \ldots\}$ and $\omega=$ $\{0,1,2, \ldots\}$. Also, $\omega$ is the first infinite cardinal.) We use the $\vec{x}$ notation throughout to represent both column and row vectors, expecting the reader to rely on the context to tell which is meant.

Theorem 1.2. Let $u, v \in \mathbb{N}$ and let $A$ be $a u \times v$ matrix with entries from $\mathbb{Q}$. The following statements are equivalent.

(a) $A$ is image partition regular.

(b) For every central subset $C$ of $\mathbb{N}$, there exists $\vec{x} \in \mathbb{N}^{v}$ such that $A \vec{x} \in C^{u}$.

(c) For every central subset $C$ of $\mathbb{N},\left\{\vec{x} \in \mathbb{N}^{v}\right.$ such that $\left.A \vec{x} \in C^{u}\right\}$ is central in $\mathbb{N}^{v}$. 
(d) For each $\vec{r} \in \mathbb{Q}^{v} \backslash\{\overrightarrow{0}\}$ there exists $b \in \mathbb{Q} \backslash\{0\}$ such that

$$
\left(\begin{array}{c}
b \vec{r} \\
A
\end{array}\right)
$$

is image partition regular.

(e) For every central subset $C$ of $\mathbb{N}$, there exists $\vec{x} \in \mathbb{N}^{v}$ such that $\vec{y}=A \vec{x} \in C^{u}$, all entries of $\vec{x}$ are distinct, and for all $i, j \in\{1,2, \ldots, u\}$, if rows $i$ and $j$ of $A$ are unequal, then $y_{i} \neq y_{j}$.

Proof. [6] Theorem 2.10].

Infinite image partition regular matrices are also of significant interest. For example, the Finite Sums Theorem (see [4, Theorem 3.15] or [7, Corollary 5.9]) is the assertion that the matrix

$$
\left(\begin{array}{cccc}
1 & 0 & 0 & \ldots \\
0 & 1 & 0 & \ldots \\
1 & 1 & 0 & \ldots \\
0 & 0 & 1 & \ldots \\
0 & 1 & 1 & \ldots \\
1 & 1 & 1 & \ldots \\
\vdots & \vdots & \vdots & \ddots
\end{array}\right)
$$

(whose rows are all vectors with entries from $\{0,1\}$ with only finitely many 1 's and not all 0 's) is image partition regular.

The fact, guaranteed by statement (d) of Theorem 1.2 that finite image partition regular matrices can be almost arbitrarily extended is very useful (and a property we would hope to maintain with infinite image partition regular matrices). Another important property was originally established by W. Deuber in 1] in terms of "( $m, p, c)$-sets". That property is an immediate consequence of Theorem 1.2(b), namely that if $A$ and $B$ are finite image partition regular matrices, then the matrix

$$
\left(\begin{array}{ll}
A & \mathbf{O} \\
\mathbf{O} & B
\end{array}\right)
$$

is also image partition regular.

The question of which infinite matrices are image partition regular seems to be significantly more complicated than the finite case. For example, it was shown in [2] that there are infinite image partition regular matrices $A$ and $B$ such that the matrix $\left(\begin{array}{ll}A & \mathbf{O} \\ \mathbf{O} & B\end{array}\right)$ is not image partition regular. (See Theorem 2.2 below.) Not many examples of infinite image partition regular matrices are known. The matrices which we shall describe now have been essentially the only known examples, all based on the Milliken-Taylor Theorem ([8, 11], or see [7, Section 18.1]) or variants thereof.

Definition 1.3. Let $\vec{a}$ be a (finite or infinite) sequence in $\mathbb{Q}$ with only finitely many nonzero entries. Then $c(\vec{a})$ is the finite sequence obtained by first deleting all occurrences of 0 and then deleting any term equal to its predecessor. A compressed sequence is a (necessarily finite) sequence $\vec{a}$ such that $c(\vec{a})=\vec{a}$.

For example, if $\vec{a}=(1,0,0,1,1,-2,0,-2,0,3,0,0,0,0, \ldots)$, then $c(\vec{a})=(1,-2$, $3)$. In [2, Theorem 2.5] it was shown that if $A$ is an $\omega \times \omega$ matrix with entries from $\omega$ (and only finitely many nonzero entries on each row) such that the compressed forms 
of all rows were equal, then $A$ is image partition regular, and it is a consequence of Corollary 3.6 below, that the same statement holds if the entries are allowed to come from $\mathbb{Z}$, provided the rightmost nonzero entries are positive.

It has not even been known if even the simplest diagonal sums are image partition regular. For example, let

$$
A=\left(\begin{array}{cccc}
1 & 1 & 0 & \cdots \\
1 & 0 & 1 & \cdots \\
0 & 1 & 1 & \cdots \\
\vdots & \vdots & \vdots & \ddots
\end{array}\right) \text { and } B=\left(\begin{array}{cccc}
1 & 2 & 0 & \cdots \\
1 & 0 & 2 & \cdots \\
0 & 1 & 2 & \cdots \\
\vdots & \vdots & \vdots & \ddots
\end{array}\right)
$$

It is an immediate consequence of Ramsey's Theorem that both $A$ and $B$ are image partition regular by way of vectors $\vec{x}$ and $\vec{z}$ without repeated terms, but it has not been known whether $\left(\begin{array}{ll}A & \mathbf{O} \\ \mathbf{O} & B\end{array}\right)$ is image partition regular in the same fashion. That is, it has not been known whether whenever $\mathbb{N}$ is finitely colored, there must exist injective sequences $\left\langle x_{n}\right\rangle_{n=0}^{\infty}$ and $\left\langle z_{n}\right\rangle_{n=0}^{\infty}$ in $\mathbb{N}$ with all sums of the forms $x_{n}+x_{m}$ and $z_{n}+2 z_{m}$ with $n<m$ in the same color class.

In Section 2 we shall present some contrasts between finite and infinite partition regular matrices and, motivated by considerations presented there, introduce the notions of centrally image partition regular matrices (those having images in any central subset of $\mathbb{N}$ ) and strongly centrally image partition regular matrices (those centrally image partition regular matrices for which distinct rows produce distinct entries of the image). It turns out that these classes do have some of the closure properties that one would like. In particular, if $A$ and $B$ are both centrally image partition regular or both strongly centrally image partition regular, then $\left(\begin{array}{ll}A & \mathbf{O} \\ \mathbf{O} & B\end{array}\right)$ has the corresponding property. It is not true that matrices whose rows have the same compressed form are centrally image partition regular. However, we show in Section 3 that matrices whose rows have the same compressed form and the same nonzero row sum are strongly centrally image partition regular, and we show that the corresponding statement for matrices with row sums of zero is not true. We obtain as a consequence, in Corollary 3.8, new results about (ordinary) image partition regularity, such as the result mentioned in the abstract.

\section{Contrasts Between Finite AND infinite MAtrices}

We take, as the principal good properties of finite partition regular matrices that we would like infinite partition regular matrices to share, the characterization of Theorem 1.2(d) and the result of [6, Corollary 2.11]. That is, we would like it to be true that whenever $A$ is an infinite image partition regular matrix and $\vec{r} \in \mathbb{Q}^{\omega} \backslash\{\overrightarrow{0}\}$ (with only finitely many nonzero entries) there should be some $b \in \mathbb{Q} \backslash\{0\}$ such that $\left(\begin{array}{c}b \vec{r} \\ A\end{array}\right)$ is image partition regular. We would also like it to be the case that whenever $A$ and $B$ are infinite image partition regular matrices, so is $\left(\begin{array}{l}A \\ \mathbf{O}\end{array}\right.$ and $B$ are $\omega \times \omega$ matrices, then $\left(\begin{array}{l}A \\ O\end{array}\right.$ blissfully ignore this and similar distinctions throughout this paper.)

We shall see in Theorems 2.1] and 2.2 that neither of our main wishes with respect to infinite image partition regular matrices can be granted.

Theorem 2.1. Let $A$ be a matrix whose rows are all rows $\vec{a} \in \mathbb{Q}^{\omega}$ with only finitely many nonzero entries such that $c(\vec{a})=(1,2)$. Let $\vec{r}=\left(\begin{array}{ccccc}1 & 0 & 0 & 0 & \ldots\end{array}\right)$. Then 
$A$ is image partition regular, but there does not exist $b \in \mathbb{Q}$ such that $\left(\begin{array}{c}b \vec{r} \\ A\end{array}\right)$ is image partition regular.

Proof. As we remarked above, that $A$ is image partition regular follows from [2, Theorem 2.5].

Suppose we have $b \in \mathbb{Q}$ such that $\left(\begin{array}{c}b \vec{r} \\ A\end{array}\right)$ is image partition regular, and notice that trivially $b>0$. Write $b=\frac{m}{n}$, where $m$ and $n$ are relatively prime positive integers.

Case 1. $m \neq 1$. Given $x \in \mathbb{N}$, let $\alpha(x)=\max \left\{t \in \omega: m^{t} \mid x\right\}$. For $i \in\{0,1\}$, let $B_{i}=\{x \in \mathbb{N}: \alpha(x) \equiv i(\bmod 2)\}$. Choose $i \in\{0,1\}$ and $\vec{x} \in \mathbb{N}^{\omega}$ such that

$$
\left(\begin{array}{c}
b \vec{r} \\
A
\end{array}\right) \vec{x} \in B_{i}^{\omega} \text {. }
$$

Then the first row says that $\frac{m}{n} x_{0} \in B_{i}$, and so $\alpha\left(\frac{m}{n} x_{0}\right) \equiv i(\bmod 2)$. Also $\alpha\left(\frac{m}{n} x_{0}\right)=$ $\alpha\left(m x_{0}\right)$, since $m$ and $n$ are relatively prime. Consequently, $\alpha\left(x_{0}\right) \not \equiv i(\bmod 2)$.

Let $s=\alpha\left(x_{0}\right)$ and pick $F \in \mathcal{P}_{f}(\mathbb{N})$ such that $m^{s+1} \mid \sum_{t \in F} x_{t}$, where $\mathcal{P}_{f}(\mathbb{N})$ is the set of finite nonempty subsets of $\mathbb{N}$. Pick $G \in \mathcal{P}_{f}(\mathbb{N})$ such that $\max F<\min G$ and $m^{s+1} \mid \sum_{t \in G} 2 x_{t}$. Then $x_{0}+\sum_{t \in F} x_{t}+\sum_{t \in G} 2 x_{t}$ is an entry of $\left(\begin{array}{c}b \vec{r} \\ A\end{array}\right) \vec{x}$ while $\alpha\left(x_{0}+\sum_{t \in F} x_{t}+\sum_{t \in G} 2 x_{t}\right)=s \not \equiv i(\bmod 2)$, a contradiction.

Case 2. $m=1$ and $n \neq 1$. Given $x \in \mathbb{N}$, let $\alpha(x)=\max \left\{t \in \omega: n^{t} \mid x\right\}$. For $i \in\{0,1\}$, let $B_{i}=\left\{x \in \mathbb{N}: \alpha(x) \equiv i(\bmod 2)\right.$. Choose $i \in\{0,1\}$ and $\vec{x} \in \mathbb{N}^{\omega}$ such that

$$
\left(\begin{array}{c}
b \vec{r} \\
A
\end{array}\right) \vec{x} \in B_{i}^{\omega} .
$$

Then the first row says that $\frac{1}{n} x_{0} \in B_{i}$, and so $\alpha\left(\frac{1}{n} x_{0}\right) \equiv i(\bmod 2)$. Consequently, $\alpha\left(x_{0}\right) \not \equiv i(\bmod 2)$. Choosing $F$ and $G$ as in Case 1 , we again obtain a contradiction.

Case 3. $b=1$. For $x \in \mathbb{N}$, let $\varphi(x)$ count the number of odd length blocks of 0's interior to the binary expansion of $x$. (More formally, if $x=\sum_{t \in F} 2^{t}$ and $k=\max F$, then $\varphi(x)=\mid\{t \in F \backslash\{k\}:(\min \{s \in F: s>t\}-t)$ is even $\} \mid$.$) For$ $j \in\{0,1,2\}$, let $B_{j}=\{x \in \mathbb{N}: \varphi(x) \equiv j(\bmod 3)\}$.

Choose $j \in\{0,1,2\}$ and $\vec{x} \in \mathbb{N}^{\omega}$ such that $\left(\begin{array}{c}\vec{r} \\ A\end{array}\right) \vec{x} \in B_{j}^{\omega}$. The first row of this equation says that $\varphi\left(x_{0}\right) \equiv j(\bmod 3)$. Choose inductively a sequence $\left\langle H_{t}\right\rangle_{t=1}^{\infty}$ in $\mathcal{P}_{f}(\mathbb{N})$ such that for each $t, \max H_{t}<\min H_{t+1}$ and, if $2^{s} \leq \sum_{i \in H_{t}} x_{i}$, then $2^{s+1} \mid \sum_{i \in H_{t+1}} x_{i}$. For each $t \in \mathbb{N}$, let $z_{t}=\sum_{i \in H_{t}} x_{i}$ and choose $F_{t} \in \mathcal{P}_{f}(\mathbb{N})$ such that $z_{t}=\sum_{i \in F_{t}} 2^{i}$. Notice that for each $t, \max F_{t}<\min F_{t+1}$.

Pick $s \in \mathbb{N}$ such that $2^{s}>x_{0}$. Choose $u<k<l$ in $\mathbb{N}$ such that $\varphi\left(x_{u}\right) \equiv \varphi\left(x_{k}\right) \equiv$ $\varphi\left(x_{l}\right)(\bmod 3), \min F_{u} \equiv \min F_{k} \equiv \min F_{l}(\bmod 2)$, and $\max F_{u} \equiv \max F_{k} \equiv$ $\max F_{l}(\bmod 2)$. Notice that $2 z_{l}+z_{k}+z_{u}$ and $2 z_{l}+z_{k}+z_{u}+x_{0}$ are both entries of $\left(\begin{array}{c}\vec{r} \\ A\end{array}\right) \vec{x}$, and so $2 z_{l}+z_{k}+z_{u} \in B_{j}$ and $2 z_{l}+z_{k}+z_{u}+x_{0} \in B_{j}$.

Now $\varphi\left(2 z_{l}+z_{k}+z_{u}\right)=\varphi\left(z_{l}\right)+\varphi\left(z_{k}\right)+\varphi\left(z_{u}\right)+1$. (Exactly one odd block of 0's is added in addition to those interior to the expansions of $2 z_{l}, z_{k}$, and $z_{u}$. It is between $z_{k}$ and $z_{u}$ if $\max F_{u} \equiv \min F_{u}(\bmod 2)$, and it is between $2 z_{l}$ and $z_{k}$ if $\max F_{u} \not \equiv$ $\left.\min F_{u}(\bmod 2).\right)$ Consequently, $\varphi\left(2 z_{l}+z_{k}+z_{u}\right) \equiv 1(\bmod 3)$, and thus $j=1$, and so $\varphi\left(x_{0}\right) \equiv 1(\bmod 3)$. Now $\varphi\left(2 z_{l}+z_{k}+z_{u}+x_{0}\right)=\varphi\left(2 z_{l}+z_{k}+z_{u}\right)+\varphi\left(x_{0}\right)+1$ or 
$\varphi\left(2 z_{l}+z_{k}+z_{u}+x_{0}\right)=\varphi\left(2 z_{l}+z_{k}+z_{u}\right)+\varphi\left(x_{0}\right)$, depending on whether or not an odd block of 0's is introduced between the expansions of $z_{u}$ and $x_{0}$. Consequently, $\varphi\left(2 z_{l}+z_{k}+z_{u}+x_{0}\right) \equiv 0(\bmod 3)$ or $\varphi\left(2 z_{l}+z_{k}+z_{u}+x_{0}\right) \equiv 2(\bmod 3)$. In either case, we have a contradiction.

Theorem 2.2. Let $\vec{b}$ be a compressed sequence with entries from $\mathbb{N}$ such that $\vec{b} \neq$ (1). Let $A$ be a matrix whose rows are all rows $\vec{a} \in \mathbb{Q}^{\omega}$ with only finitely many nonzero entries such that $c(\vec{a})=\vec{b}$. Let $B$ be the finite sums matrix.

(a) The matrices $A$ and $B$ are image partition regular.

(b) There is a subset $C$ of $\mathbb{N}$ that is a member of every idempotent in $\beta \mathbb{N}$ (and is thus, in particular, central) such that for no $\vec{x} \in \mathbb{N}^{\omega}$ does one have $A \vec{x} \in C^{\omega}$.

(c) The matrix $\left(\begin{array}{ll}A & \mathbf{O} \\ \mathbf{O} & B\end{array}\right)$ is not image partition regular.

Proof. Note that $B$ is a matrix whose rows are all rows $\vec{a} \in \mathbb{Q}^{\omega}$ with only finitely many nonzero entries such that $c(\vec{a})=(1)$. That $A$ and $B$ are image partition regular follows from [2. Theorem 2.5].

By [2, Theorem 3.14], there exist $C_{1}$ and $C_{2}$ such that $\mathbb{N}=C_{1} \cup C_{2}$ and there does not exist $\vec{x} \in \mathbb{N}^{\omega}$ with $A \vec{x} \in C_{1}^{\omega}$ and there does not exist $\vec{y} \in \mathbb{N}^{\omega}$ with $B \vec{y} \in C_{2}^{\omega}$, and thus $\left(\begin{array}{ll}A & \mathbf{O}\end{array}\right)$ is not image partition regular. By [7, Theorem 5.8], $C_{2}$ is not a member of any idempotent in $\beta \mathbb{N}$, and thus $C_{1}$ is a member of every idempotent in $\beta \mathbb{N}$.

By way of contrast with Theorem 2.2(c), we see that infinite image partition regular matrices can be extended by finite ones. (We are grateful to V. Rödl for providing us with this result and its proof.)

Lemma 2.3. Let $A$ and $B$ be finite and infinite image partition regular matrices respectively (with rational coefficients). Then $\left(\begin{array}{ll}A & \mathbf{O} \\ \mathbf{O} & B\end{array}\right)$ is image partition regular.

Proof. Assume that $A$ is a $u \times v$ matrix. Let $r \in \mathbb{N}$ and let $\mathbb{N}$ be $r$-colored by $\varphi$. Let (by compactness) $n$ be large enough so that whenever $\{1,2, \ldots, n\}$ is $r$-colored, there exists $\vec{x} \in \mathbb{N}^{v}$ such that the entries of $A \vec{x}$ are monochrome.

Now color $\mathbb{N}$ with $r^{n}$ colors via $\psi$, where $\psi(x)=\psi(y)$ if and only if for all $t \in\{1,2, \ldots, n\}, \varphi(t x)=\varphi(t y)$. Pick $\vec{y} \in \mathbb{N}^{\omega}$ such that the entries of $B \vec{y}$ are monochrome with respect to $\psi$. Pick an entry $a$ of $B \vec{y}$ and define $\gamma:\{1,2, \ldots$, $n\} \rightarrow\{1,2, \ldots, r\}$ by $\gamma(i)=\varphi(i a)$. Pick $\vec{x} \in \mathbb{N}^{v}$ such that the entries of $A \vec{x}$ are monochrome with respect to $\gamma$. Pick an entry $i$ of $A \vec{x}$ and let $j=\gamma(i)$.

Let $\vec{z}=\left(\begin{array}{c}a \vec{x} \\ i \vec{y}\end{array}\right)$. We claim that for any row $\vec{w}$ of $\left(\begin{array}{ll}A & \mathbf{O} \\ \mathbf{O} & B\end{array}\right), \varphi(\vec{w} \cdot \vec{z})=j$. To see this, first assume that $\vec{w}$ is a row of $\left(\begin{array}{ll}A \quad \mathbf{O}\end{array}\right)$, so that $\vec{w}=\vec{s} \frown \overrightarrow{0}$, where $\vec{s}$ is a row of $A$. Then $\vec{w} \cdot \vec{z}=a(\vec{s} \cdot \vec{x})$ and $j=\gamma(\vec{s} \cdot \vec{x})=\varphi(a(\vec{s} \cdot \vec{x}))=\varphi(\vec{w} \cdot \vec{z})$.

Next assume that $\vec{w}$ is a row of ( $\left.\begin{array}{ll}\mathbf{O} & B\end{array}\right)$, so that $\vec{w}=\overrightarrow{0}-\vec{s}$, where $\vec{s}$ is a row of $B$. Then $\vec{w} \cdot \vec{z}=i(\vec{s} \cdot \vec{y})$. Now $\psi(\vec{s} \cdot \vec{y})=\psi(a)$, and so $\varphi(i(\vec{s} \cdot \vec{y}))=\varphi(i a)=\gamma(i)=$ $j$.

Definition 2.4. Let $A$ be a finite or infinite matrix with entries from $\mathbb{Q}$. Then $C(A)=\{p \in \beta \mathbb{N}$ : for every $P \in p$, there exists $\vec{x}$ with entries from $\mathbb{N}$ such that all the entries of $A \vec{x}$ are in $P\}$.

Lemma 2.5. Let $A$ be a matrix with entries from $\mathbb{Q}$.

(a) The set $C(A)$ is compact, and $C(A) \neq \emptyset$ if and only if $A$ is image partition regular. 
(b) If $A$ is a finite image partition regular matrix, then $C(A)$ is a subsemigroup of $\beta \mathbb{N}$.

Proof. (a) The fact that $C(A)$ is compact is trivial, as is the fact that $A$ is image partition regular if $C(A) \neq \emptyset$. Assume that $A$ is image partition regular and let

$$
\begin{aligned}
\mathcal{C}=\{B \subseteq \mathbb{N}: & \text { for every } \vec{x} \text { with entries from } \mathbb{N} \text { and the same number of entries } \\
& \text { as } A \text { has columns, some entry of } A \vec{x} \text { is in } B\} .
\end{aligned}
$$

We claim that $\mathcal{C}$ has the finite intersection property. To see this, suppose instead that we have $n \in \mathbb{N}$ and $B_{1}, B_{2}, \ldots, B_{n} \in \mathcal{C}$ with $\bigcap_{i=1}^{n} B_{i}=\emptyset$. Then $\mathbb{N}=\bigcup_{i=1}^{n}\left(\mathbb{N} \backslash B_{i}\right)$, and so there are some $i \in\{1,2, \ldots, n\}$ and some $\vec{x}$ with all entries of $A \vec{x}$ in $\mathbb{N} \backslash B_{i}$, contradicting the fact that $B_{i} \in \mathcal{C}$.

Since $\mathcal{C}$ has the finite intersection property, pick $p \in \beta \mathbb{N}$ with $\mathcal{C} \subseteq p$. To see that $p \in C(A)$, let $P \in p$. If there were no $\vec{x}$ with entries from $\mathbb{N}$ such that all entries of $A \vec{x}$ are in $P$, we would have $\mathbb{N} \backslash P \in \mathcal{C} \subseteq p$, a contradiction.

(b) Let $u, v \in \mathbb{N}$ be such that $A$ is a $u \times v$ matrix. We have $C(A) \neq \emptyset$ by (a). Let $p, q \in C(A)$ and let $B \in p+q$. Then $C=\{y \in \mathbb{N}:-y+B \in q\} \in p$; so pick $\vec{x} \in \mathbb{N}^{v}$ such that $\vec{y}=A \vec{x} \in C^{u}$. Then $D=\bigcap_{i=1}^{u}\left(-y_{i}+B\right) \in q$; so pick $\vec{z} \in \mathbb{N}^{v}$ such that $A \vec{z} \in D^{u}$. Then $A(\vec{x}+\vec{z}) \in B^{u}$.

The set $C(A)$ need not be a semigroup if $A$ is an infinite image partition regular matrix, as can be seen from Theorem [2.2, wherein the matrix $A$ is image partition regular, but $C(A)$ contains no idempotents.

Corollary 2.6. Let $\mathcal{F}$ denote the set of finite image partition regular matrices over $\mathbb{Q}$. If $B$ is an infinite partition regular matrix, then $C(B) \cap \bigcap_{A \in \mathcal{F}} C(A) \neq \emptyset$.

Proof. Let $A_{1}, A_{2}, \cdots, A_{n}$ be a finite number of elements of $\mathcal{F}$. Let

$$
M=\left(\begin{array}{ccccc}
A_{1} & \mathbf{O} & \cdots & \mathbf{O} & \mathbf{O} \\
\mathbf{O} & A_{2} & \cdots & \mathbf{O} & \mathbf{O} \\
\vdots & \vdots & \ddots & \vdots & \vdots \\
\mathbf{O} & \mathbf{O} & \cdots & A_{n} & \mathbf{O} \\
\mathbf{O} & \mathbf{O} & \cdots & \mathbf{O} & B
\end{array}\right)
$$

By Lemma 2.3. $M$ is image partition regular, and so

$$
\emptyset \neq C(M) \subseteq C(B) \cap \bigcap_{i=1}^{n} C\left(A_{i}\right) .
$$

Our claim now follows from compactness.

We saw in Theorem 2.2(b) that the characterization of Theorem 1.2 (b) need not be valid for infinite image partition regular matrices. This leads us to hope that perhaps matrices with this stronger property are better behaved.

Definition 2.7. Let $A$ be an $\omega \times \omega$ matrix. Then $A$ is centrally image partition regular if and only if whenever $C$ is a central set in $\mathbb{N}$, there exists $\vec{x} \in \mathbb{N}^{\omega}$ such that $A \vec{x} \in C^{\omega}$.

It is trivial that whenever $A$ and $B$ are centrally image partition regular matrices, then so is $\left(\begin{array}{ll}A & \mathbf{O} \\ \mathbf{O} & B\end{array}\right)$. Unfortunately, our other desired characteristic does not hold. 
Proposition 2.8. There is an $\omega \times 2$ centrally image partition regular matrix $A$ with entries from $\mathbb{Q}$ such that there does not exist $b \in \mathbb{Q}$ making $\left({ }^{-b}{ }_{A} b\right)$ image partition regular. In fact, there do not exist $b \in \mathbb{Q}$ and $\vec{x}=\left(\begin{array}{c}x_{0} \\ x_{1}\end{array}\right) \in \mathbb{N}^{2}$ with $\left(\begin{array}{cc}-b & b \\ A\end{array}\right) \vec{x} \in \mathbb{N}^{\omega}$.

Proof. Let

$$
A=\left(\begin{array}{cc}
\frac{1}{2} & \frac{1}{2} \\
\frac{1}{3} & \frac{2}{3} \\
\frac{1}{4} & \frac{3}{4} \\
\frac{1}{5} & \frac{4}{5} \\
\vdots & \vdots
\end{array}\right) .
$$

Given any central set $C$, pick $a \in C$ and let $\vec{x}=\left(\begin{array}{l}a \\ a\end{array}\right)$. Then all entries of $A \vec{x}$ are equal to $a$.

Suppose that we have $b \in \mathbb{Q}$ and $\vec{x}=\left(\begin{array}{l}x_{0} \\ x_{1}\end{array}\right) \in \mathbb{N}^{2}$ with $\left(\begin{array}{cc}-b & b \\ A\end{array}\right) \vec{x} \in \mathbb{N}^{\omega}$.

Since $-b \cdot x_{0}+b \cdot x_{1} \in \mathbb{N}$, we have that $x_{0} \neq x_{1}$. Pick $n \in \mathbb{N}$ such that $\left|x_{0}-x_{1}\right|<n$. Then $\frac{1}{n} \cdot x_{0}+\frac{n-1}{n} \cdot x_{1} \in \mathbb{N}$ while $\frac{1}{n} \cdot x_{0}+\frac{n-1}{n} \cdot x_{1}=x_{1}+\frac{x_{0}-x_{1}}{n}$. But $x_{1} \in \mathbb{N}$ and $0<\frac{\left|x_{0}-x_{1}\right|}{n}<1$, a contradiction.

We now turn our attention to the characterization of Theorem 1.2 (e). Considering the matrix of Proposition 2.8, which could only produce entries in $\mathbb{N}$ if the entries of $\vec{x}$ were equal, it seems reasonable to ask that the entries of $\vec{x}$ be required to be distinct. However, we see now that this also would do no good. (We also see that requiring the entries of the matrix to come from $\mathbb{Z}$ rather than $\mathbb{Q}$ is of no use either.)

Proposition 2.9. There is an $\omega \times 2$ matrix $A$ with entries from $\mathbb{Z}$ with the property that whenever $C$ is a central set in $\mathbb{N}$, there exists $x_{0} \neq x_{1}$ such that all entries of $A\left(\begin{array}{l}x_{0} \\ x_{1}\end{array}\right)$ are in $C$, but there does not exist $b \in \mathbb{Q}$ making $\left({ }^{2 b}{ }_{A}^{-b}\right)$ image partition regular.

Proof. Let

$$
A=\left(\begin{array}{cc}
1 & 0 \\
3 & -1 \\
5 & -2 \\
\vdots & \vdots \\
2 n+1 & -n \\
\vdots & \vdots
\end{array}\right)
$$

and let $\left\{D_{1}, D_{2}\right\}$ be any partition of $\mathbb{N}$ such that neither $D_{1}$ nor $D_{2}$ contains infinite arithmetic progressions.

To see that $A$ has the first claimed property, let $C$ be a central set and pick $x_{0} \in C$. Let $x_{1}=2 x_{0}$. Then all entries of $A \vec{x}$ are equal to $x_{0}$.

Suppose that we have $b \in \mathbb{Q}, i \in\{1,2\}$, and $\vec{x}=\left(\begin{array}{c}x_{0} \\ x_{1}\end{array}\right) \in \mathbb{N}^{2}$ with

$$
\left(\begin{array}{cc}
2 b & -b \\
A
\end{array}\right) \vec{x} \in D_{i}^{\omega}
$$


Then $2 b x_{0}-b x_{1}>0 ;$ so $2 x_{0} \neq x_{1}$. Let $a=\frac{x_{1}}{x_{0}}$. Then the $n^{\text {th }}$ entry of $A \vec{x}$ is $x_{0}+(2-a) \cdot x_{0} \cdot n$. This tells us in fact that $a<2$, and consequently $D_{i}$ contains an infinite arithmetic progression, a contradiction.

In view of Proposition 2.9, we turn our attention to the other half of the characterization of Theorem $1.2(\mathrm{e})$.

Definition 2.10. Let $A$ be an $\omega \times \omega$ matrix. Then $A$ is strongly centrally image partition regular if and only if whenever $C$ is a central set in $\mathbb{N}$, there exists $\vec{x} \in \mathbb{N}^{\omega}$ such that $\vec{y}=A \vec{x} \in C^{\omega}$ and for all $i, j \in \omega$, if rows $i$ and $j$ of $A$ are unequal, then $y_{i} \neq y_{j}$.

There is a simple necessary condition for a matrix to be strongly centrally image partition regular.

Theorem 2.11. Let $A$ be a strongly centrally image partition regular matrix without repeated rows. Then for each $k \in \mathbb{N},\left\{i:\right.$ for all $\left.j \geq k, a_{i, j}=0\right\}$ is finite.

Proof. Suppose instead that $\left\{i\right.$ : for all $\left.j \geq k, a_{i, j}=0\right\}$ is infinite. Then by discarding the other rows we may presume that $A$ is an $\omega \times k$ matrix. Let $D=$ $\left\{\vec{x} \in \mathbb{N}^{k}\right.$ : all entries of $A \vec{x}$ are distinct $\}$. Enumerate $D$ as $\left\langle\vec{x}^{(n)}\right\rangle_{n=1}^{\infty}$. Inductively choose distinct $y_{n}$ and $z_{n}$ in $A \vec{x}^{(n)}$, with $\left\{y_{n}, z_{n}\right\} \cap\left(\left\{y_{t}: t \in\{1,2, \ldots, n-1\}\right\} \cup\left\{z_{t}\right.\right.$ : $t \in\{1,2, \ldots, n-1\}\})=\emptyset$ if $n>1$. Let $C=\left\{y_{n}: n \in \mathbb{N}\right\}$. Then there is no $\vec{x} \in D$ with $A \vec{x} \in C^{\omega}$, and no $\vec{x} \in D$ with $A \vec{x} \in(\mathbb{N} \backslash C)^{\omega}$.

We shall see in Theorem 3.9 that there is a strongly centrally image partition regular matrix $A$ such that there is no $b \in \mathbb{Q} \backslash\{0\}$ for which $\left(\begin{array}{c}b \vec{r} \\ A\end{array}\right)$ is image partition regular, where $\vec{r}=\left(\begin{array}{ccccc}1 & 0 & 0 & 0 & \ldots\end{array}\right)$.

We shall see in Corollary 2.14 that the strongly centrally image partition regular matrices do maintain one of the properties that we desire. First we shall have need of the following algebraic result, which we think is of interest in its own right. (The information about the minimal left and minimal right ideals involved is not needed here. But it is interesting algebraically and costs us little additional effort.) Notice that since, by [7, Theorem 2.7] $R \cap L$ is a group, $p$ is the unique idempotent of $R \cap L$.

Theorem 2.12. Let $p$ be a minimal idempotent in $(\beta \mathbb{N},+)$ and let $L$ and $R$ be respectively the minimal left and minimal right ideals of $(\beta \mathbb{N},+)$ with $p \in L \cap R$. Then for each $C \in p$, there are $2^{\mathfrak{c}}$ minimal idempotents in $L \cap \bar{C}$ and $2^{\mathfrak{c}}$ minimal idempotents in $R \cap \bar{C}$.

Proof. Let $C \in p$, let $C^{\star}=\{x \in C:-x+C \in p\}$, and notice that, by [7, Lemma 4.14], for each $x \in C^{\star}$ we have $-x+C^{\star} \in p$. For each $m \in \omega$, let

$$
S_{m}=2^{m} \mathbb{N} \cap C^{\star} \cap \bigcap\left\{-k+C^{\star}: k \in C^{\star} \cap\{1,2, \ldots, m\}\right\} .
$$

Let $V=\bigcap_{m \in \mathbb{N}} \overline{S_{m}}$. For every $m \in \mathbb{N}$ we have $2^{m} \mathbb{N} \in p$ (by [7, Lemma 6.6]), and so $S_{m} \in p$. Thus $p \in V$.

We show that $V$ is a subsemigroup of $\beta \mathbb{N}$, using [7, Theorem 4.20]. So, let $m \in \mathbb{N}$ and let $n \in S_{m}$. It suffices to show that $n+S_{m+n} \subseteq S_{m}$. Let $r \in S_{m+n}$. Certainly $n+r \in 2^{m} \mathbb{N}$. Since $n \in C^{\star} \cap\{1,2, \ldots, m+n\}, n+r \in C^{\star}$. Let $k \in C^{\star} \cap\{1,2, \ldots, m\}$. Then $n \in-k+C^{\star}$; so $k+n \in C^{\star} \cap\{1,2, \ldots, m+n\}$, and thus $r \in-(k+n)+C^{\star}$, so that $n+r \in-k+C^{\star}$, as required. 
Since $p \in V$, we have by [7, Theorem 6.32] that $V$ contains a copy of $\mathbb{H}=$ $\bigcap_{n=1}^{\infty} \overline{\mathbb{N} 2^{n}}$. (This copy is guaranteed to be both an algebraic and topological copy, via the same function, but here we only care about the fact that it is an algebraic copy.) By [7 Theorem 6.9], $(\beta \mathbb{N},+)$ has $2^{\mathfrak{c}}$ minimal left ideals. So there is a set $W \subseteq \beta \mathbb{N}$ of idempotents such that $|W|=2^{\mathfrak{c}}$ and, whenever $u$ and $v$ are distinct members of $W, u+v \neq u$ and $v+u \neq v$. Since by [7, Lemma 6.6], $W \subseteq \mathbb{H}$ and $V$ contains a copy of $\mathbb{H}$, we have a set $E \subseteq V$ of idempotents such that $|E|=2^{\mathfrak{c}}$ and, whenever $u$ and $v$ are distinct members of $E, u+v \neq u$ and $v+u \neq v$.

By [7. Corollary 6.20], if $u$ and $v$ are distinct members of $E$, then $(\beta \mathbb{N}+u) \cap$ $(\beta \mathbb{N}+v)=\emptyset$; so, in particular, $(V+u) \cap(V+v)=\emptyset$. For each $u \in E$, pick an idempotent $\alpha_{u} \in(p+V) \cap(V+u)$ with $\alpha_{u}$ minimal in $V$. (By [7], Corollary 2.6 and Theorem 2.7], $p+V$ contains a minimal right ideal of $V$ and $V+u$ contains a minimal left ideal of $V$, and the intersection of a minimal right ideal of $V$ with a minimal left ideal of $V$ is a group. Let $\alpha_{u}$ be the identity of this group.) Then $\left\{\alpha_{u}: u \in E\right\}$ is a set of $2^{\mathfrak{c}}$ idempotents in $p+V \subseteq R$, each minimal in $V$. Since $p \in V \cap K(\beta \mathbb{N})$, we have that $K(V)=V \cap K(\beta \mathbb{N})$ by [7, Theorem 1.65], so that each $\alpha_{u}$ is minimal in $\beta \mathbb{N}$.

Now we verify the assertion about $L$. For each $x \in \mathbb{N}$ define $\operatorname{supp}(x) \in \mathcal{P}_{f}(\omega)$ by $x=\sum_{t \in \operatorname{supp}(x)} 2^{t}$. Inductively choose a sequence $\left\langle r_{n}\right\rangle_{n=1}^{\infty}$ in $\mathbb{N}$ such that, for each $n \in \mathbb{N}, r_{n} \in S_{n}$ and $\max \operatorname{supp}\left(r_{n}\right)<\min \operatorname{supp}\left(r_{n+1}\right)$. Let $X=\left\{r_{n}: n \in \mathbb{N}\right\}$ and note that $\bar{X} \cap \mathbb{N}^{*}=\beta \mathbb{N} \backslash \mathbb{N} \subseteq V$. Note also that, since $S_{n} \subseteq \mathbb{N}^{n}, V \subseteq \mathbb{H}$. Define $f: \mathbb{N} \rightarrow \omega$ by $f(n)=\min \operatorname{supp}(n)$, and let $\tilde{f}: \beta \mathbb{N} \rightarrow \beta \omega$ be its continuous extension. Notice that if $x \in \beta \mathbb{N}$ and $q \in \mathbb{H}$, then $\widetilde{f}(x+q)=\widetilde{f}(x)$. (To see this it suffices to show that the continuous functions $\tilde{f} \circ \rho_{q}$ and $\tilde{f}$ agree on $\mathbb{N}$. If $n \in \mathbb{N}$ and $m=f(n)+1$, then $\tilde{f} \circ \lambda_{n}$ is constantly equal to $f(n)$ on $\mathbb{N} 2^{m}$, and so $\widetilde{f}(n+q)=f(n)$.

For each $y \in \widetilde{f}\left[\bar{X} \cap \mathbb{N}^{*}\right]$ we know that $\{q \in V: \widetilde{f}(q)=y\}$ is a right ideal of $V$. So pick an idempotent $\delta_{y} \in\{q \in V: \widetilde{f}(q)=y\} \cap L$ that is minimal in $V$. Each $\delta_{y}$ is minimal in $V$, hence in $\beta \mathbb{N}$, and if $y \neq z$, then $\{q \in V: \widetilde{f}(q)=y\} \cap\{q \in V$ : $\widetilde{f}(q)=z\}=\emptyset$. It thus suffices to show that $\left|\widetilde{f}\left[\bar{X} \cap \mathbb{N}^{*}\right]\right|=2^{\mathfrak{c}}$. To see this, let $v$ be any nonprincipal ultrafilter on $\left\{f\left(r_{n}\right): n \in \mathbb{N}\right\}$ (of which there are $2^{\mathfrak{c}}$ ). For $A \in v$, let $B(A)=\left\{r_{n}: f\left(r_{n}\right) \in A\right\}$. Then $\{B(A): A \in v\}$ has the finite intersection property; so pick $q \in \beta \mathbb{N}$ with $\{B(A): A \in v\} \subseteq q$. Since $\bigcap_{A \in v} B(A)=\emptyset, q \in \mathbb{N}^{*}$. Also, $\widetilde{f}(q)=v$.

Corollary 2.13. Let $C$ be a central set in $\mathbb{N}$. Then there exists a sequence $\left\langle C_{n}\right\rangle_{n=1}^{\infty}$ of pairwise disjoint central sets in $\mathbb{N}$ with $\bigcup_{n=1}^{\infty} C_{n} \subseteq C$.

Proof. By Theorem 2.12, the set of minimal idempotents in $\bar{C}$ is infinite, hence contains an infinite strongly discrete subset. (Alternatively, there are two minimal idempotents in $\bar{C}$, so that $C$ can be split into two central sets, $C_{1}$ and $D_{1}$. Then $D_{1}$ can be split into two central sets, $C_{2}$ and $D_{2}$, and so on.) 
Corollary 2.14. For each $n \in \mathbb{N}$, let $A_{n}$ be a strongly centrally image partition regular matrix. Then the matrix

$$
M=\left(\begin{array}{cccc}
A_{1} & \mathbf{O} & \mathbf{O} & \ldots \\
\mathbf{O} & A_{2} & \mathbf{O} & \ldots \\
\mathbf{O} & \mathbf{O} & A_{3} & \ldots \\
\vdots & \vdots & \vdots & \ddots
\end{array}\right)
$$

is also strongly centrally image partition regular.

Proof. Let $C$ be a central set and choose by Corollary 2.13 a sequence $\left\langle C_{n}\right\rangle_{n=1}^{\infty}$ of pairwise disjoint central sets in $\mathbb{N}$ with $\bigcup_{n=1}^{\infty} C_{n} \subseteq C$. For each $n \in \mathbb{N}$ choose $\vec{x}^{(n)} \in \mathbb{N}^{\omega}$ such that $\vec{y}^{(n)}=A_{n} \vec{x}^{(n)} \in C_{n}^{\omega}$ and, if rows $i$ and $j$ of $A_{n}$ are unequal, $y_{i}^{(n)} \neq y_{j}^{(n)}$. Let

$$
\vec{z}=\left(\begin{array}{c}
\vec{x}^{(1)} \\
\vec{x}^{(2)} \\
\vdots
\end{array}\right) .
$$

Then all entries of $M \vec{z}$ are in $C$, and entries from distinct rows are unequal.

Of course Corollary 2.14 remains valid if "strongly centrally image partition regular" is replaced by "centrally image partition regular". The same proof applies, and one does not need to introduce the pairwise disjoint central sets, which were required to guarantee that the entries of $M \vec{z}$ from distinct rows were distinct.

\section{Constant Row sums}

Notice that trivially, if $A$ is an $\omega \times \omega$ matrix with entries from $\mathbb{Q}$ and there is some positive $m \in \mathbb{Q}$ such that each row of $A$ sums to $m$, then $A$ is centrally image partition regular. (Given a central set $C$, simply pick $d \in \mathbb{N}$ such that $d m \in C$, which one can do because for each $n \in \mathbb{N}, \mathbb{N} n$ is a member of every idempotent by 7. Lemma 6.6]. Then let $x_{i}=d$ for each $i \in \omega$.) We also saw in Theorem 2.2(b) that if $\vec{b}$ is a compressed sequence with entries from $\omega$ such that $\vec{b} \neq(1)$ and $A$ is a matrix whose rows are all rows $\vec{a} \in \mathbb{Q}^{\omega}$ with only finitely many nonzero entries such that $c(\vec{a})=\vec{b}$, then $A$ is not centrally image partition regular.

We shall show in this section (in Theorem 3.7) that if $A$ is a matrix with entries from $\mathbb{Z}$ with finitely many nonzero entries in each row such that the compressed forms of all rows of $A$ are the same and all rows of $A$ have the same nonzero sum, then $A$ is strongly centrally image partition regular. We shall also show (in Corollary 3.15) that a matrix with the same compressed form for all rows and zero sum for each row need not be centrally image partition regular.

We show now that any matrix with constant positive row sums and a limited number of patterns in any finite set of columns can be extended at will. That the restriction on the number of patterns in a finite set of columns is needed can be seen by considering the matrix of Proposition 2.8. We shall see in Theorem 3.9 that we cannot extend the following theorem to the case in which $m \in \mathbb{Z}$.

In Theorem 3.1 we talk of adding finitely many rows, rather than adding rows one at a time as in Theorem[1.2(d), because we cannot simply iterate the procedure. (If the row sums of $A$ are all $m$ and the sum of row $\vec{r}$ is not 0 , one can multiply $\vec{r}$ by $b$ so that its sum is $m$ and iterate. However, in the interesting cases, some or all of the added rows will sum to 0 .) 
Theorem 3.1. Let $k \in \mathbb{N}$, let $m \in \mathbb{Q}$ with $m>0$, and let $A$ be an $\omega \times \omega$ matrix with entries from $\mathbb{Q}$ such that

(i) the sum of each row of $A$ is $m$, and

(ii) for each $l \in \omega,\left\{\left\langle a_{i, 0}, a_{i, 1}, \ldots, a_{i, l}\right\rangle: i \in \omega\right\}$ is finite.

Let $\vec{r}^{(1)}, \vec{r}^{(2)}, \ldots, \vec{r}^{(k)} \in \mathbb{Q}^{\omega} \backslash\{\overrightarrow{0}\}$ such that each $\vec{r}^{(i)}$ has only finitely many nonzero entries. Then there exist $b_{1}, b_{2}, \ldots, b_{k} \in \mathbb{Q} \backslash\{0\}$ such that

$$
\left(\begin{array}{c}
b_{1} \vec{r}^{(1)} \\
b_{2} \vec{r}^{(2)} \\
\vdots \\
b_{k} \vec{r}^{(k)} \\
A
\end{array}\right)
$$

is centrally image partition regular.

Proof. Pick $l \in \mathbb{N}$ such that for every $j \in\{1,2, \ldots, k\}$ and every $i \geq l$ we have $r_{i}^{(j)}=0$. For each $j \in\{1,2, \ldots, k\}$, let $s^{(j)}=\left\langle r_{0}^{(j)}, r_{1}^{(j)}, \ldots, r_{l}^{(j)}\right\rangle$. Enumerate

$$
\left\{\left\langle a_{i, 0}, a_{i, 1}, \ldots, a_{i, l-1}\right\rangle: i \in \omega\right\}
$$

as $\vec{w}^{(0)}, \vec{w}^{(1)}, \ldots, \vec{w}^{(u)}$. For each $i \in\{0,1, \ldots, u\}$, let $d_{i}=m-\sum_{j=0}^{l-1} w_{j}^{(i)}$. Let $E$ be the $(u+1) \times(l+1)$ matrix with entries

$$
e_{i, j}=\left\{\begin{array}{cl}
w_{j}^{(i)} & \text { if } j \in\{0,1, \ldots, l-1\} \\
d_{i} & \text { if } j=l
\end{array}\right.
$$

Then $E$ has constant row sums, so is image partition regular. By applying Theorem 1.2 (d) $u+1$ times, pick $b_{1}, b_{2}, \ldots, b_{k} \in \mathbb{Q} \backslash\{0\}$ such that the matrix

$$
H=\left(\begin{array}{c}
b_{1} \vec{s}^{(1)} \\
b_{2} \vec{s}^{(2)} \\
\vdots \\
b_{k} \vec{s}^{(k)} \\
E
\end{array}\right)
$$

is image partition regular, hence, by Theorem [1.2(b), centrally image partition regular.

Let $C$ be a central set and pick $\left\langle z_{0}, z_{1}, \ldots, z_{l}\right\rangle \in \mathbb{N}^{l+1}$ such that $H \vec{z} \in C^{u+1}$. For $n \in\{0,1, \ldots, l-1\}$, let $x_{n}=z_{n}$. For $n \in\{l, l+1, l+2, \ldots\}$, let $x_{n}=z_{l}$. Then

$$
\left(\begin{array}{c}
b_{1} \vec{r}^{(1)} \\
b_{2} \vec{r}^{(2)} \\
\vdots \\
b_{k} \vec{r}^{(k)} \\
A
\end{array}\right) \vec{x} \in C^{\omega} .
$$

We see now that matrices with constant positive row sums need not be strongly centrally image partition regular.

Proposition 3.2. Let $A$ be an $\omega \times \omega$ matrix whose rows are all those rows with entries from $\{0,1,2\}$ with exactly one 1 and exactly one 2 (and no repeated rows). 
While $A$ is centrally image partition regular, it is not strongly centrally image partition regular. In fact, there is a two-cell partition $\left\{D_{0}, D_{1}\right\}$ of $\mathbb{N}$ such that there do not exist $t \in\{0,1\}$ and $\vec{x} \in \mathbb{N}^{\omega}$ with $\vec{y}=A \vec{x} \in D_{t}^{\omega}$ and all entries of $\vec{y}$ distinct.

Proof. For $x \in \mathbb{N}$, let $f(x)=\max \operatorname{supp}(x)$, where $x=\sum_{t \in \operatorname{supp}(x)} 2^{t}$. (Then $2^{f(x)} \leq x<2^{f(x)+1}$.) For $t \in\{0,1\}$, let $D_{t}=\{x \in \mathbb{N}: f(x) \equiv t(\bmod 2)\}$. Suppose that we have $t \in\{0,1\}$ and $\vec{x} \in \mathbb{N}^{\omega}$ with $\vec{y}=A \vec{x} \in D_{t}^{\omega}$ and all entries of $\vec{y}$ distinct. Then also all entries of $\vec{x}$ are distinct. (If $i \neq j$, then $2 x_{i}+x_{j}$ and $x_{i}+2 x_{j}$ are distinct entries of $\vec{y}$.)

Pick $k<l<m$ in $\omega$ such that $f\left(x_{k}\right)+3<f\left(x_{l}\right)<f\left(x_{m}\right)$. If we had $i<j$ in $\{k, l, m\}$ such that $x_{j}<2^{f\left(x_{j}\right)+1}-2 x_{i}$, then we would have $f\left(x_{j}+2 x_{i}\right)=f\left(x_{j}\right)$ while $f\left(2 x_{j}+x_{i}\right)=f\left(x_{j}\right)+1$, a contradiction. Thus for $i<j$ in $\{k, l, m\}$ we have $x_{j} \geq 2^{f\left(x_{j}\right)+1}-2 x_{i}$ and $f\left(x_{j}+2 x_{i}\right)=f\left(x_{j}\right)+1$. In particular,

$$
x_{l} \geq 2^{f\left(x_{l}\right)+1}-2 x_{k}
$$

and

$$
x_{m} \geq 2^{f\left(x_{m}\right)+1}-2 x_{k} .
$$

If we had $2 x_{m} \geq 2^{f\left(x_{m}\right)+2}-x_{l}$, then we would have $f\left(2 x_{m}+x_{l}\right)=f\left(x_{m}\right)+2 \not \equiv$ $f\left(x_{m}+2 x_{l}\right)(\bmod 2)$, again a contradiction. Thus, using $(*)$ and $(* *)$, we have

$$
2^{f\left(x_{m}\right)+2}-4 x_{k} \leq 2 x_{m}<2^{f\left(x_{m}\right)+2}-x_{l} \leq 2^{f\left(x_{m}\right)+2}-2^{f\left(x_{l}\right)+1}+2 x_{k},
$$

so that $x_{l}<2^{f\left(x_{l}\right)+1}<6 x_{k}<8 x_{k}<2^{f\left(x_{k}\right)+4} \leq 2^{f\left(x_{l}\right)} \leq x_{l}$, a contradiction.

Notice that any finite set of rows of the matrix $A$ in Proposition 3.2 forms a finite image partition regular matrix (after throwing away columns with all zeroes). Thus by Theorem 1.2 (e), given any central set $C$ and any $n \in \mathbb{N}$, there must exist $\vec{x} \in \mathbb{N}^{\omega}$ such that the first $n$ entries of $A \vec{x}$ are distinct and lie in $C$.

It is a consequence of Theorem 3.7 below that if the matrix $A$ defined in Proposition 3.2 is modified by requiring that the occurrence of 1 comes before the occurrence of 2 on each row (or vice versa), then the resulting matrix is strongly centrally image partition regular. The proof of Theorem 3.7 uses a quite general construction, which we present now.

Recall that if $D$ is a discrete space, $p \in \beta D, X$ is a topological space, $y \in X$, and $f: D \rightarrow X$, then $p$ - $\lim _{s \in D} f(s)=y$ if and only if for every neighborhood $U$ of $y$ in $X,\{s \in D: f(s) \in U\} \in p$. See [7, Section 3.5] for a presentation of the basic properties of these limits.

Given a sequence $\left\langle x_{k}\right\rangle_{k=0}^{\infty}$ in a semigroup $(S, \cdot)$, we write

$$
F P\left(\left\langle x_{k}\right\rangle_{k=0}^{\infty}\right)=\left\{\prod_{k \in F} x_{k}: F \in \mathcal{P}_{f}(\omega)\right\}
$$

for the set of finite products of terms of the sequence. If the operation is denoted by + , then we write $F S\left(\left\langle x_{k}\right\rangle_{k=0}^{\infty}\right)=\left\{\sum_{k \in F} x_{k}: F \in \mathcal{P}_{f}(\omega)\right\}$.

Theorem 3.3. Let $S$ and $T$ be discrete spaces, let $n \in \mathbb{N}$, and let $f: S^{n} \rightarrow T$. Define $g: \beta S \rightarrow \beta T$ by

$$
g(p)=p-\lim _{s_{1} \in S} p-\lim _{s_{2} \in S} \ldots p-\lim _{s_{n} \in S} f\left(s_{1}, s_{2}, \ldots, s_{n}\right) .
$$

Let $p \in S^{*}=\beta S \backslash S$, let $h: \bigcup_{k=1}^{\infty} S^{k} \rightarrow p$, let $Q \in g(p)$, and let $A \in p$. 
(1) There exists a sequence $\left\langle x_{k}\right\rangle_{k=0}^{\infty}$ in $A$ such that whenever $r_{1}<r_{2}<\ldots<r_{n}$ we have $f\left(x_{r_{1}}, x_{r_{2}}, \ldots, x_{r_{n}}\right) \in Q$, and for each $k \in \mathbb{N}, x_{k} \in h\left(x_{0}, x_{1}, \ldots, x_{k-1}\right)$.

(2) If $(S, \cdot)$ is a semigroup and $p$ is an idempotent, then the sequence $\left\langle x_{k}\right\rangle_{k=0}^{\infty}$ can be chosen so that, in addition to the above conclusions, $F P\left(\left\langle x_{k}\right\rangle_{k=0}^{\infty}\right) \subseteq A$ and whenever $F_{1}, F_{2}, \ldots, F_{n}$ are finite nonempty subsets of $\omega$ with $\max F_{k}<\min F_{k+1}$ for every $k \in\{1,2, \ldots, n-1\}$, one has $f\left(\Pi_{t \in F_{1}} x_{t}, \Pi_{t \in F_{2}} x_{t}, \ldots, \Pi_{t \in F_{n}} x_{t}\right) \in Q$.

Proof. Let $P=\left\{z \in A: p\right.$ - $\lim _{s_{2} \in S} p-\lim _{s_{3} \in S} \ldots p$ - $\lim _{s_{n} \in S} f\left(z, s_{2}, s_{3}, \ldots, s_{n}\right) \in$ $\bar{Q}\}$. Note that $P \in p$. For $k \in\{1,2, \ldots, n-2\}$ and $y_{1}, y_{2}, \ldots, y_{k} \in S$, let $P_{y_{1}, y_{2}, \ldots, y_{k}}$ $=\left\{z \in S: p-\lim _{s_{k+2} \in S} p-\lim _{s_{k+3} \in S} \ldots p-\lim _{s_{n} \in S} f\left(y_{1}, y_{2}, \ldots, y_{k}, z, s_{k+2}, \ldots, s_{n}\right)\right.$ $\in \bar{Q}\}$ and for $y_{1}, y_{2}, \ldots, y_{n-1} \in S$, let $P_{y_{1}, y_{2}, \ldots, y_{n-1}}=\left\{z \in S: f\left(y_{1}, y_{2}, \ldots\right.\right.$, $\left.\left.y_{n-1}, z\right) \in Q\right\}$.

Notice that, if $y \in P$, then $P_{y} \in p$ and, if $k \in\{1,2, \ldots, n-2\}$ and $y_{k+1} \in$ $P_{y_{1}, y_{2}, \ldots, y_{k}}$, then $P_{y_{1}, y_{2}, \ldots, y_{k+1}} \in p$.

To establish the conclusions in (1), pick $x_{0} \in P$, let $m \in \omega$, and assume that we have chosen $x_{0}, x_{1}, \ldots, x_{m} \in P$ such that

(i) for each $k \in\{1,2, \ldots, m\}, x_{k} \in h\left(x_{1}, x_{2}, \ldots, x_{k-1}\right)$, and

(ii) for each $k \in\{1,2, \ldots, m\}$, if $k \leq n$ and $0 \leq r_{1}<r_{2}<\ldots<r_{k} \leq m$, then $x_{r_{k}} \in P_{x_{r_{1}}, x_{r_{2}}, \ldots, x_{r_{k-1}}}$.

For $k \in\{1,2, \ldots, n-1\}$, let

$$
V_{k}=\left\{\left(x_{r_{1}}, x_{r_{2}}, \ldots, x_{r_{k}}\right): 0 \leq r_{1}<r_{2}<\ldots<r_{k} \leq m\right\}
$$

Choose

$$
x_{m+1} \in P \cap h\left(x_{0}, x_{1}, \ldots, x_{m}\right) \cap \bigcap_{k=1}^{\min \{n-1, m+1\}} \bigcap_{\left(y_{1}, y_{2}, \ldots, y_{k}\right) \in V_{k}} P_{y_{1}, y_{2}, \ldots, y_{k}} .
$$

The induction hypotheses guarantee that the set on the right is a member of $p$, and is therefore nonempty.

Now, to verify the conclusions in (2), assume that $(S, \cdot)$ is a semigroup and $p=p \cdot p$. For any $B \in p$, let $B^{\star}=\left\{x \in B: x^{-1} B \in p\right\}$, where $x^{-1} B=\{y \in$ $S: x \cdot y \in B\}$. Then $B^{\star} \in p$ and, by [7, Lemma 4.14], whenever $x \in B^{\star}$, one has $x^{-1} B^{\star} \in p$.

Choose $x_{0} \in P^{\star}$. Let $m \in \mathbb{N}$, and assume that we have chosen $x_{0}, x_{1}, \ldots, x_{m}$ such that

(i) for each $k \in\{1,2, \ldots, m\}, x_{k} \in h\left(x_{1}, x_{2}, \ldots, x_{k-1}\right)$,

(ii) if $\emptyset \neq F \subseteq\{0,1, \ldots, m\}$, then $\prod_{t \in F} x_{t} \in P^{\star}$, and

(iii) if $k \in\{2,3, \ldots, n\}$ and $F_{1}, F_{2}, \ldots, F_{k} \in \mathcal{P}_{f}(\{1,2, \ldots, m\})$ with $\max F_{j}<$ $\min F_{j+1}$ for each $j \in\{1,2, \ldots, k-1\}$, then

$$
\prod_{t \in F_{k}} x_{t} \in\left(P_{\Pi_{t \in F_{1}} x_{t}, \Pi_{t \in F_{2}} x_{t}, \ldots, \Pi_{t \in F_{k-1}} x_{t}}\right)^{\star} .
$$

For $r \in\{0,1, \ldots, m\}$, let $E_{r}=\left\{\prod_{t \in F} x_{t}: \emptyset \neq F \subseteq\{r, r+1, \ldots, m\}\right\}$, and for $k \in\{1,2, \ldots, n-1\}$ and $r \in\{0,1, \ldots, m\}$, let

$$
\begin{aligned}
W_{k, r}= & \left\{\left(\prod_{t \in F_{1}} x_{t}, \prod_{t \in F_{2}} x_{t}, \ldots, \prod_{t \in F_{k}} x_{t}\right): F_{1}, F_{2}, \ldots, F_{k} \in \mathcal{P}_{f}(\{0,1, \ldots, r\})\right. \\
& \text { and } \left.\max F_{j}<\min F_{j+1} \text { for every } j \in\{1,2, \ldots, k-1\}\right\} .
\end{aligned}
$$

Note that $W_{k, r} \neq \emptyset$ if and only if $k \leq m+1$

Hypothesis (ii) tells us that if $y \in E_{0}$ (equivalently if $(y) \in W_{1, m}$ ), then $y \in P^{\star}$, so that $y^{-1} P^{\star} \in p$ and $P_{y} \in p$.

Hypothesis (iii) tells us that whenever $k \in\{2,3, \ldots, n-1\}$ and $\left(y_{1}, y_{2}, \ldots, y_{k}\right) \in$ $W_{k, m}$, one has $y_{k} \in P_{y_{1}, y_{2}, \ldots, y_{k-1}}$, so that $P_{y_{1}, y_{2}, \ldots, y_{k}} \in p$. 
Hypothesis (iii) also tells us that if $r \in\{0,1, \ldots, m-1\}, k \in\{1,2, \ldots, n-1\}$, $\left(y_{1}, y_{2}, \ldots, y_{k}\right) \in W_{k, r}$, and $z \in E_{r+1}$, then $z \in\left(P_{y_{1}, y_{2}, \ldots, y_{k}}\right)^{\star}$, and thus

$$
z^{-1}\left(P_{y_{1}, y_{2}, \ldots, y_{k}}\right)^{\star} \in p \text {. }
$$

Thus we may choose

$$
\begin{aligned}
x_{m+1} \in & h\left(x_{0}, x_{1}, \ldots, x_{m}\right) \cap P^{\star} \cap \bigcap_{y \in E_{0}} y^{-1} P^{\star} \\
& \cap \bigcap_{k=1}^{\min \{m+1, n-1\}} \bigcap_{\left(y_{1}, y_{2}, \ldots, y_{k}\right) \in W_{k, m}}\left(P_{y_{1}, y_{2}, \ldots, y_{k}}\right)^{\star} \\
& \cap \bigcap_{r=0}^{m-1} \bigcap_{k=1}^{\min \{r+1, n-1\}} \bigcap_{\left(y_{1}, y_{2}, \ldots, y_{k}\right) \in W_{k, r}} \bigcap_{z \in E_{r+1}} z^{-1}\left(P_{y_{1}, y_{2}, \ldots, y_{k}}\right)^{\star},
\end{aligned}
$$

because this set is a member of $p$ and is therefore nonempty.

Hypothesis (i) holds directly. To verify hypothesis (ii), let $\emptyset \neq F \subseteq\{1,2, \ldots$, $m+1\}$ with $m+1 \in F$. If $F=\{m+1\}$, then $\prod_{t \in F} x_{t}=x_{m+1} \in P^{\star}$. Otherwise, let $G=F \backslash\{m+1\}$ and let $y=\prod_{t \in G} x_{t}$. Then $y \in E_{0}$, and so $x_{m+1} \in y^{-1} P^{\star}$, and thus $\prod_{t \in F} x_{t} \in P^{\star}$.

To verify hypothesis (iii), let $k \in\{2,3, \ldots, n\}$ and let $F_{1}, F_{2}, \ldots, F_{k} \in \mathcal{P}_{f}(\{1,2$, $\ldots, m+1\})$ with $\max F_{j}<\min F_{j+1}$ for each $j \in\{1,2, \ldots, k-1\}$ and with $m+1 \in$ $F_{k}$. If $F_{k}=\{m+1\}$, then we have

$$
\prod_{t \in F_{k}} x_{t}=x_{m+1} \in\left(P_{\Pi_{t \in F_{1}} x_{t}, \Pi_{t \in F_{2}} x_{t}, \ldots, \Pi_{t \in F_{k-1}} x_{t}}\right)^{\star} .
$$

So assume that $F_{k} \neq\{m+1\}$, let $G=F \backslash\{m+1\}$, and let $r=\min G-1$. Let $z=$ $\Pi_{t \in G} x_{t}$, and for $j \in\{1,2, \ldots, k-1\}$ let $y_{j}=\prod_{t \in F_{j}} x_{t}$. Then $\left(y_{1}, y_{2}, \ldots, y_{k-1}\right) \in$ $W_{k-1, r}$ and $z \in E_{r+1}$, so that $\prod_{t \in F_{k}} x_{t}=z \cdot x_{m+1} \in\left(P_{y_{1}, y_{2}, \ldots, y_{k-1}}\right)^{\star}$, as required.

As we have previously remarked, in [2, Theorem 2.5] it was shown that if $A$ is an $\omega \times \omega$ matrix with entries from $\omega$ (and only finitely many nonzero entries on each row) such that the compressed forms of all rows were equal, then $A$ is image partition regular. We extend this result now to allow negative entries. Notice that in the following lemma and beyond, if $p \in \beta \mathbb{N}$ and $a \in \mathbb{Z}$, then the product $a \cdot p$ refers to multiplication in the semigroup $(\beta \mathbb{Z}, \cdot)$. In particular, if $a \in \mathbb{N}, a \cdot p$ is not the sum of $p$ with itself $a$ times. (If, as here, $p=p+p$, that sum is just $p$.)

In our remaining results we shall be assuming that the entries of our matrices come from $\mathbb{Z}$ rather than $\mathbb{Q}$. This is a convenient, but not essential, restriction because we shall also be assuming that the compressed forms of all rows are equal, so that any such matrix with rational entries can be turned into one with integer entries by multiplying by a constant. Since multiplying a central set by a constant produces another central set [6, Lemma 2.1], the corresponding results hold.

Lemma 3.4. Let $\left\langle a_{1}, a_{2}, \ldots, a_{k}\right\rangle$ be a sequence in $\mathbb{Z} \backslash\{0\}$. Let $m=\max \left\{\left|a_{j}\right|\right.$ : $j \in\{1,2, \ldots, k\}\}$, let $p$ be an idempotent in $\beta \mathbb{N}$, and let $q=a_{1} \cdot p+a_{2} \cdot p+\ldots+$ $a_{k} \cdot p \in \beta \mathbb{Z}$. If $A \in q$ and $P \in p$, then there is a sequence $\left\langle x_{n}\right\rangle_{n=0}^{\infty}$ in $\mathbb{N}$ such that $x_{n+1}>2 m \cdot \sum_{t=0}^{n} x_{t}$ for each $n \in \omega, F S\left(\left\langle x_{n}\right\rangle_{n=0}^{\infty}\right) \subseteq P$ and $\left\{\sum_{t=1}^{k} a_{t} \cdot \sum_{n \in F_{t}} x_{n}\right.$ : $F_{1}, F_{2}, \ldots, F_{k} \in \mathcal{P}_{f}(\mathbb{N})$ and $\max F_{t}<\min F_{t+1}$ for $\left.t \in\{1,2, \ldots, k-1\}\right\} \subseteq A$.

Proof. Define $h: \bigcup_{n=1}^{\infty} \mathbb{N}^{n} \rightarrow p$ and $f: \mathbb{N}^{k} \rightarrow \mathbb{Z}$ by $h\left(x_{0}, x_{1}, \ldots, x_{n-1}\right)=\{z \in \mathbb{N}$ : $\left.z>2 m \cdot \sum_{t=1}^{n-1} x_{t}\right\}$ and $f\left(y_{1}, y_{2}, \ldots, y_{k}\right)=a_{1} \cdot y_{1}+a_{2} \cdot y_{2}+\ldots+a_{k} \cdot y_{k}$. Then

$$
p-\lim _{y_{1} \in \mathbb{N}} p-\lim _{y_{2} \in \mathbb{N}} \ldots p-\lim _{y_{k} \in \mathbb{N}} f\left(y_{1}, y_{2}, \ldots, y_{k}\right)=a_{1} \cdot p+a_{2} \cdot p+\ldots+a_{k} \cdot p .
$$

So Theorem 3.3 applied to the semigroup $(\mathbb{N},+)$ yields the desired conclusion. 
We observe now that the sequence produced in Lemma 3.4 satisfies a strong uniqueness of sums property.

Lemma 3.5. Let $\left\langle a_{1}, a_{2}, \ldots, a_{k}\right\rangle$ be a compressed sequence in $\mathbb{Z} \backslash\{0\}$, let $m=$ $\max \left\{\left|a_{j}\right|: j \in\{1,2, \ldots, k\}\right\}$, and let $\left\langle x_{n}\right\rangle_{n=0}^{\infty}$ be a sequence in $\mathbb{N}$ such that $x_{n+1}>$ $2 m \cdot \sum_{t=0}^{n} x_{t}$ for each $n \in \omega$. Then whenever $F_{1}, F_{2}, \ldots, F_{k}, G_{1}, G_{2}, \ldots, G_{k} \in$ $\mathcal{P}_{f}(\mathbb{N}), \max F_{t}<\min F_{t+1}$ and $\max G_{t}<\min G_{t+1}$ for $t \in\{1,2, \ldots, k-1\}$, and $\sum_{t=1}^{k} a_{t} \cdot \sum_{n \in F_{t}} x_{n}=\sum_{t=1}^{k} a_{t} \cdot \sum_{n \in G_{t}} x_{n}$, one must have $F_{t}=G_{t}$ for each $t \in\{1,2$, $\ldots, k\}$.

Proof. Suppose instead that we have some $F_{1}, F_{2}, \ldots, F_{k}, G_{1}, G_{2}, \ldots, G_{k} \in \mathcal{P}_{f}(\mathbb{N})$ such that $\max F_{t}<\min F_{t+1}$ and $\max G_{t}<\min G_{t+1}$ for $t \in\{1,2, \ldots, k-1\}$, $\sum_{t=1}^{k} a_{t} \cdot \sum_{n \in F_{t}} x_{n}=\sum_{t=1}^{k} a_{t} \cdot \sum_{n \in G_{t}} x_{n}$, but $F_{t} \neq G_{t}$ for some $t \in\{1,2, \ldots, k\}$. Pick the largest $l \in\{1,2, \ldots, k\}$ such that $F_{l} \neq G_{l}$. Then $\sum_{t=1}^{l} a_{t} \cdot \sum_{n \in F_{t}} x_{n}=$ $\sum_{t=1}^{l} a_{t} \cdot \sum_{n \in G_{t}} x_{n}$. Let $r=\max \left(F_{l} \Delta G_{l}\right)$. By subtracting any larger terms from both sides of the last equation, we may presume that $r=\max \left(F_{l} \cup G_{l}\right)$. Assume without loss of generality that $r \in F_{l}$. We may also assume that $a_{l}>0$. (Otherwise, multiply both sides by -1 .) Then

$$
\begin{aligned}
\sum_{t=1}^{l} a_{t} \cdot \sum_{n \in F_{t}} x_{n} & \geq a_{r} x_{r}-\sum_{n=1}^{r-1} m x_{n} \\
& >\sum_{n=1}^{r-1} m x_{n} \\
& \geq \sum_{t=1}^{l} a_{t} \cdot \sum_{n \in G_{t}} x_{n}
\end{aligned}
$$

a contradiction.

Corollary 3.6. Let $\left\langle a_{1}, a_{2}, \ldots, a_{k}\right\rangle$ be a compressed sequence in $\mathbb{Z} \backslash\{0\}$ with $a_{k}>$ 0 . Let $M$ be a matrix, with finitely many nonzero entries in each row, such that the compressed form of each row is $\left\langle a_{1}, a_{2}, \ldots, a_{k}\right\rangle$. Then $M$ is image partition regular. Indeed, given any idempotent $p \in \beta \mathbb{N}$ and any function $h: \bigcup_{t \in \mathbb{N}} \mathbb{N}^{t} \rightarrow p$, if $q=a_{1} \cdot p+a_{2} \cdot p+\ldots+a_{k} \cdot p$, then $q \in \bigcap_{n=1}^{\infty} \overline{n \mathbb{N}}$, and, for any $A \in q$ and any $P \in p$, there is an increasing sequence $\vec{x} \in \mathbb{N}^{\omega}$ such that $F S\left(\left\langle x_{n}\right\rangle_{n=0}^{\infty}\right) \subseteq P, x_{n+1} \in$ $h\left(x_{i_{1}}, x_{i_{2}}, \cdots, x_{i_{m}}\right)$ for every $n \in \mathbb{N}$ and every choice of $0 \leq i_{1}<i_{2} \ldots<i_{m} \leq n$, $M \vec{x} \in A^{\omega}$, and entries corresponding to distinct rows are distinct.

Proof. Let $p$ be an idempotent in $\beta \mathbb{N}$ and let $q=a_{1} \cdot p+a_{2} \cdot p+\ldots+a_{k} \cdot p$. Let $T=\bigcap_{n=1}^{\infty} \overline{n \mathbb{Z}}$. We show first that $q \in T \cap \beta \mathbb{N}=\bigcap_{n=1}^{\infty} \overline{n \mathbb{N}}$. By [7, Lemma 6.6], $p \in T$. Since, by [7, Theorems 2.15 and 2.17], $T$ is an ideal of $(\beta \mathbb{Z}, \cdot)$, we have that each $a_{i} \cdot p \in T$. By [7, Exercise 2.3.2] $T$ is a subsemigroup of $(\beta \mathbb{Z},+)$, and so $q \in T$. Since $a_{k}>0$ we have that $a_{k} \cdot p \in \mathbb{N}^{*}$, and so $q \in \beta \mathbb{N}$ by [7, Exercise 4.3.5].

Let $A \in q$ and $P \in p$, and choose a sequence $\left\langle x_{n}\right\rangle_{n=0}^{\infty}$ as guaranteed by Lemma 3.4 for $A$ and $P$. If $\vec{x}=\left\langle x_{n}\right\rangle_{n=0}^{\infty}$, then all the entries of $M \vec{x}$ are in $A$. By Lemma [3.5, entries that correspond to distinct rows are distinct.

Notice that if $\vec{x}$ is as guaranteed by Corollary 3.6 and $\vec{z}$ is a subsequence of $\vec{x}$, then also $M \vec{z} \in A^{\omega}$, and entries that correspond to distinct rows are distinct, because any entry of $M \vec{z}$ is also an entry of $M \vec{x}$. 
We remark that the possibility of choosing $x_{n+1} \in h\left(x_{i_{1}}, x_{i_{2}}, \cdots, x_{i_{m}}\right)$, guaranteed by Corollary [3.6, yields nontrivial information about simple finite matrices. For example, let $A=\left(\begin{array}{ll}1 & 2 \\ 0 & 1\end{array}\right)$. Then $A$ is image partition regular. Color $\mathbb{N}$ according to the parity of $\max (\operatorname{supp}(n))$. (Recall that $\operatorname{supp}(n) \in \mathcal{P}_{f}(\omega)$ is defined for $n \in \mathbb{N}$ by $n=\sum_{i \in \operatorname{supp}(n)} 2^{i}$.) We cannot choose $\vec{x} \in \mathbb{N}^{2}$ such that $\min \left(\operatorname{supp}\left(x_{2}\right)\right)>\max \left(\operatorname{supp}\left(x_{1}\right)\right)$ and the entries of $A \vec{x}$ are monochrome. However, if $B=\left(\begin{array}{lll}1 & 1 & 2 \\ 0 & 1 & 2\end{array}\right)$, then, defining $h(x)=\{y \in \mathbb{N}: \min (\operatorname{supp}(y))>\max (\operatorname{supp}(x))\}$ and $h(x, y)=\{z \in \mathbb{N}: \min (\operatorname{supp}(z))>\max (\operatorname{supp}(y))\}$, Corollary 3.6 guarantees that, in any finite coloring of $\mathbb{N}$, there exists $\vec{x} \in \mathbb{N}^{3}$ such that $\min \left(\operatorname{supp}\left(x_{i+1}\right)\right)>$ $\max \left(\operatorname{supp}\left(x_{i}\right)\right)$ if $i \in\{1,2\}$ and the entries of $B \vec{x}$ are monochrome.

Theorem 3.7. Let $k \in \mathbb{N}$, let $\left\langle a_{1}, a_{2}, \ldots, a_{k}\right\rangle$ be a compressed sequence in $\mathbb{Z} \backslash\{0\}$ with $a_{k}>0$, and let $m \in \mathbb{Z} \backslash\{0\}$. Let $M$ be a matrix, with finitely many nonzero entries in each row, such that

(i) the compressed form of each row is $\left\langle a_{1}, a_{2}, \ldots, a_{k}\right\rangle$, and

(ii) the sum of each row is $m$.

Then $M$ is strongly centrally image partition regular.

Proof. Let

$$
\begin{aligned}
L=\{q \in \beta \mathbb{N}: & \text { for every } A \in q \text { and every } k \in \mathbb{N}, \text { there exists } \\
& \vec{x} \in\{k+1, k+2, k+3, \ldots\}^{\omega} \text { such that } M \vec{x} \in A^{\omega} \text { and } \\
& \text { entries corresponding to distinct rows of } M \text { are distinct }\} .
\end{aligned}
$$

It is obvious that $L$ is closed. By Corollary 3.6 $L \cap|m| \cdot \beta \mathbb{N} \neq \emptyset$.

We claim that $L \cap|m| \cdot \beta \mathbb{N}$ is a left ideal of $|m| \cdot \beta \mathbb{N}$. To this end, let $q \in L \cap|m| \cdot \beta \mathbb{N}$. It suffices to show that $|m| \cdot \mathbb{N}+q \subseteq L \cap|m| \cdot \beta \mathbb{N}$. We have immediately that $|m| \cdot \mathbb{N}+q \subseteq|m| \cdot \beta \mathbb{N}$. To see that $|m| \cdot \mathbb{N}+q \subseteq L$, let $n \in \mathbb{N}$, let $A \in|m| \cdot n+q$, and let $k \in \mathbb{N}$. Pick $\vec{x} \in\{k+n+1, k+n+2, k+n+3, \ldots\}^{\omega}$ such that $\vec{y}=M \vec{x} \in(-|m| \cdot n+A)^{\omega}$ and the entries of $\vec{y}$ corresponding to distinct rows are distinct. Let $s=\frac{|m|}{m}$ and for each $i \in \omega$ let $z_{i}=s n+x_{i}$. Then each $z_{i}>k$. Let $\vec{w}=M \vec{z}$. Then for each $j \in \omega$ we have that $w_{j}=|m| \cdot n+y_{j} \in A$, and entries of $\vec{w}$ corresponding to distinct rows of $M$ are distinct.

Let $C$ be a central set in $\mathbb{N}$, and pick a minimal idempotent $r$ in $\beta \mathbb{N}$ such that $C \in r$. Let $p=\frac{1}{a_{k}} \cdot r$ (multiplication in $\beta \mathbb{Q}_{d}$, where $\mathbb{Q}_{d}$ is the set $\mathbb{Q}$ with the discrete topology). It is routine to check that $p \in \beta \mathbb{N}, p+p=p$, and $a_{k} \cdot p=r$. Let $q=a_{1} \cdot p+a_{2} \cdot p+\ldots+a_{k} \cdot p$. By Corollary [3.6, $q \in L \cap|m| \cdot \beta \mathbb{N}$. Since $a_{k} \cdot p=r$, it follows that $q \in \beta \mathbb{Z}+r=\beta \mathbb{Z}+r+r \subseteq \beta \mathbb{N}+r$ (the last inclusion by 7 , Exercise 4.3.5]). Since $q \in|m| \cdot \beta \mathbb{N}$ and $r \in|m| \cdot \beta \mathbb{N}$, we have that $q \in|m| \cdot \beta \mathbb{N}+r$.

Now $r \in|m| \cdot \beta \mathbb{N} \cap K(\beta \mathbb{N})$; so by [7, Theorem 1.65], $r \in K(|m| \cdot \beta \mathbb{N})$. Thus, by 7, Theorem 2.9], $|m| \cdot \beta \mathbb{N}+r$ is a minimal left ideal of $|m| \cdot \beta \mathbb{N}$. Since

$$
q \in(L \cap|m| \cdot \beta \mathbb{N}) \cap(|m| \cdot \beta \mathbb{N}+r),
$$

we have $|m| \cdot \beta \mathbb{N}+r \subseteq L$, and so $r=r+r \in|m| \cdot \beta \mathbb{N}+r \subseteq L$.

Observe that, if all terms of $\vec{a}$ come from $\mathbb{N}$, then the number of entries of any row of a matrix $M$ as in Theorem 3.7 is limited. However, this need not be the case if one or more entries are negative. We shall see in Theorem 3.14 that the requirement that $m \neq 0$ cannot be eliminated. (We saw in Theorem [2.2(b) that the restriction on the row sums cannot simply be omitted.) 
Consider the following consequence of Theorem 3.7 Let

$$
M=\left(\begin{array}{cccc}
-2 & 1 & 0 & \ldots \\
-2 & 0 & 1 & \ldots \\
0 & -2 & 1 & \ldots \\
\vdots & \vdots & \vdots & \ddots
\end{array}\right)
$$

a matrix whose rows have somewhere a single -2 followed somewhere by a single 1. Even though matrices with constant positive row sums are trivially centrally image partition regular via a constant vector $\vec{x}$, it does not seem to be trivial that the matrix $M$ is even centrally image partition regular, while Theorem 3.7 tells us that it is in fact strongly centrally image partition regular.

As we promised earlier, we obtain, as a consequence of our consideration of strongly centrally image partition regular matrices, new results about ordinary image partition regular matrices. (The last result mentioned in the abstract is the instance of Corollary 3.8 for which $\vec{a}=\langle 1\rangle, \vec{b}=\langle 1,2\rangle, m=2$, and $n=3$.)

Corollary 3.8. Let $k, s \in \mathbb{N}$, let $\vec{a}=\left\langle a_{1}, a_{2}, \ldots, a_{k}\right\rangle$ and $\vec{b}=\left\langle b_{1}, b_{2}, \ldots, b_{s}\right\rangle$ be compressed sequences in $\mathbb{Z} \backslash\{0\}$ with $a_{k}>0$ and $b_{s}>0$, let $m, n \in \mathbb{Z} \backslash\{0\}$, and assume that there exist $\vec{r}, \vec{t} \in \mathbb{Z}^{\omega}$ such that $c(\vec{r})=\vec{a}, c(\vec{t})=\vec{b}, \sum_{j=0}^{\infty} r_{j}=m$, and $\sum_{j=0}^{\infty} t_{j}=n$. Let $q \in \mathbb{N}$ and let $\mathbb{N}=\bigcup_{i=0}^{q} C_{i}$. Then there exist $i \in\{1,2, \ldots, q\}$ and injective sequences $\left\langle x_{n}\right\rangle_{n=0}^{\infty}$ and $\left\langle z_{n}\right\rangle_{n=0}^{\infty}$ in $\mathbb{N}$ such that, for every $\vec{r}$ and $\vec{g}$ in $\mathbb{Z}^{\omega}$ with only finitely many nonzero entries, if $c(\vec{r})=\vec{a}, c(\vec{g})=\vec{b}, \sum_{j=0}^{\infty} r_{j}=m$, and $\sum_{j=0}^{\infty} g_{j}=n$, then $\sum_{j=0}^{\infty} r_{j} \cdot x_{j} \in C_{i}, \sum_{j=0}^{\infty} g_{j} \cdot z_{j} \in C_{i}$, and $\sum_{j=0}^{\infty} r_{j} \cdot x_{j} \neq$ $\sum_{j=0}^{\infty} g_{j} \cdot z_{j}$.

Proof. Let $M$ and $N$ be matrices with finitely many nonzero entries in each row such that

(a) the compressed form of each row of $M$ is $\vec{a}$;

(b) the sum of each row of $M$ is $m$;

(c) all rows with compressed form $\vec{a}$ and sum $m$ occur in $M$;

(d) the compressed form of each row of $N$ is $\vec{b}$;

(e) the sum of each row of $N$ is $n$; and

(f) all rows with compressed form $\vec{b}$ and sum $n$ occur in $N$.

Then by Theorem 3.7 and Corollary 2.14, the matrix $\left(\begin{array}{cc}M & \mathrm{O} \\ \mathrm{O} & N\end{array}\right)$ is strongly centrally image partition regular. So pick $i \in\{1,2, \ldots, q\}$ such that $C_{i}$ is central, and pick $\vec{x}$ and $\vec{z}$ in $\mathbb{N}^{\omega}$ such that all entries of $\left(\begin{array}{cc}M & \mathbf{O} \\ \mathbf{O} & N\end{array}\right)\left(\begin{array}{c}\vec{x} \\ \vec{z}\end{array}\right)$ are in $C_{i}$ and entries corresponding to distinct rows of $\left(\begin{array}{ll}M & \mathbf{O} \\ \mathbf{O} & N\end{array}\right)$ are distinct. Then one has immediately that for any row $\vec{r}$ of $M$ and any row $\vec{g}$ of $N, \sum_{u=0}^{\infty} r_{u} \cdot x_{u} \neq \sum_{u=0}^{\infty} g_{u} \cdot z_{u}$. To see, for example, that $\vec{x}$ is injective, let $j<l$ in $\omega$ and pick $t_{0}, t_{1}, \ldots, t_{v} \in \mathbb{Z} \backslash\{0\}$ such that $c\left(\left\langle t_{0}, t_{1}, \ldots, t_{v}\right\rangle\right)=\vec{a}$ and $\sum_{u=0}^{v} t_{u}=m$. Define $\vec{r}$ and $\vec{g}$ in $\mathbb{Z}^{\omega}$ by $r_{j}=g_{l}=t_{0}$, $r_{l+u}=g_{l+u}=t_{u}$ for $u \in\{1,2, \ldots, v\}$ (if any), and all other entries of $\vec{r}$ and $\vec{g}$ are equal to 0 . Then $\vec{r}$ and $\vec{g}$ are distinct rows of $m$. So $\sum_{u=0}^{\infty} r_{u} \cdot x_{u} \neq \sum_{u=0}^{\infty} g_{u} \cdot x_{u}$, and thus $x_{j} \neq x_{l}$.

We see now that strongly centrally image partition regular matrices need not have one of our desired properties, namely the analogue of Theorem 1.2 (d). 
Proposition 3.9. Let $\vec{r}=\left(\begin{array}{ccccc}1 & 0 & 0 & 0 & \ldots\end{array}\right)$. There is an $\omega \times \omega$ matrix $M$ with entries from $\mathbb{Z}$ such that the compressed form of each row is $(-2,1)$ and the sum of each row is -1 (so $M$ is strongly centrally image partition regular), but there is no $b \in \mathbb{Q} \backslash\{0\}$ such that the matrix $\left(\begin{array}{c}b \vec{r} \\ M\end{array}\right)$ is image partition regular.

Proof. Let

$$
M=\left(\begin{array}{cccc}
-2 & 1 & 0 & \ldots \\
-2 & 0 & 1 & \ldots \\
0 & -2 & 1 & \ldots \\
\vdots & \vdots & \vdots & \ddots
\end{array}\right)
$$

as described above. Suppose that $\left(\begin{array}{c}b \vec{r} \\ M\end{array}\right)$ is image partition regular for some $b \in \mathbb{Q} \backslash\{0\}$. This implies that $b>0$. Let $b=\frac{k}{l}$, where $k, l \in \mathbb{N}$. Let $r$ be a prime number satisfying $r>k+2 l$.

Each $n \in \mathbb{N}$ can be expressed uniquely as $n=\sum_{i \in \omega} a_{i} r^{i}$, where each $i \in$ $\{0,1,2, \ldots, r-1\}$. Let $\operatorname{supp}_{r}(n)=\left\{i \in \omega: a_{i} \neq 0\right\}$ and $m(n)=\min \left(\operatorname{supp}_{r}(n)\right)$. We define $f: \mathbb{N} \rightarrow\{1,2, \ldots, r-1\}$ by $f(n)=a_{m(n)}$.

Choose $\vec{x} \in \mathbb{N}^{\omega}$ and $c \in\{1,2, \ldots, r-1\}$ such that $\left(\begin{array}{c}b \vec{r} \\ M\end{array}\right) \vec{x} \in C^{\omega}$, where $C=f^{-1}[\{c\}]$. Then $b x_{0} \in C$ and $-2 x_{m}+x_{n} \in C$ whenever $n, m \in \omega$ with $m<n$. Let $d=f\left(x_{0}\right)$.

We claim that $m\left(x_{n}\right) \leq m\left(x_{0}\right)$ for every $n \in \mathbb{N}$. To see this, observe that $m\left(x_{n}\right)>m\left(x_{0}\right)$ implies that $f\left(-2 x_{0}+x_{n}\right)=-2 d$ in $\mathbb{Z}_{r}$. Since $f\left(b x_{0}\right)=\frac{k}{l} d$ in $\mathbb{Z}_{r}$, it follows that $-2 d=\frac{k}{l} d$ in $\mathbb{Z}_{r}$ and $k+2 l=0$ in $\mathbb{Z}_{r}$, a contradiction.

Thus, by the pigeonhole principle, there exist $s, t \in \mathbb{N}$ such that $t>s, m\left(x_{t}\right)=$ $m\left(x_{s}\right)$ and $f\left(x_{t}\right)=f\left(x_{s}\right)=e$, say. Then $f\left(-2 x_{s}+x_{t}\right)=-e=c$ in $\mathbb{Z}_{r}$.

We consider two cases:

(i) If $m\left(x_{s}\right)=m\left(2 x_{0}\right)$, then $f\left(-2 x_{0}+x_{s}\right)=-2 d+e=c$ in $\mathbb{Z}_{r}$, and so $d=-c$ in $\mathbb{Z}_{r}$. We also have $\frac{k}{l} d=c$ in $\mathbb{Z}_{r}$, and thus $k+l=0$ in $\mathbb{Z}_{r}$, a contradiction.

(ii) If $m\left(x_{s}\right)<m\left(2 x_{0}\right)$, then $f\left(-2 x_{0}+x_{s}\right)=e$. So $e=-e$ in $\mathbb{Z}_{r}$, again a contradiction.

The fact that the row sums in Proposition 3.9 were negative is needed to prevent the image partition regularity of $\left(\begin{array}{c}b \vec{r} \\ M\end{array}\right)$, as we shall see now. We do not know whether matrices with fixed compressed form and fixed positive row sum can necessarily be arbitrarily extended to strongly centrally image partition regular matrices.

Corollary 3.10. Let $k, l, m \in \mathbb{N}$, let $\left\langle a_{1}, a_{2}, \ldots, a_{k}\right\rangle$ be a compressed sequence in $\mathbb{Z} \backslash\{0\}$, and let $A$ be an $\omega \times \omega$ matrix such that

(i) the compressed form of each row is $\vec{a}$, and

(ii) the sum of each row of $A$ is $m$. 
Let $\vec{r}^{(1)}, \vec{r}^{(2)}, \ldots, \vec{r}^{(l)} \in \mathbb{Q}^{\omega} \backslash\{\overrightarrow{0}\}$ such that each $\vec{r}^{(i)}$ has only finitely many nonzero entries. Then there exist $b_{1}, b_{2}, \ldots, b_{k} \in \mathbb{Q} \backslash\{0\}$ such that

$$
\left(\begin{array}{c}
b_{1} \vec{r}^{(1)} \\
b_{2} \vec{r}^{(2)} \\
\vdots \\
b_{k} \vec{r}^{(l)} \\
A
\end{array}\right)
$$

is centrally image partition regular.

Proof. The matrix $A$ satisfies the hypotheses of Theorem 3.1 .

We note now that the requirement in Theorem 3.7 that $a_{k}>0$ is essential.

Theorem 3.11. Let $k \in \mathbb{N}$, let $\left\langle a_{1}, a_{2}, \ldots, a_{k}\right\rangle$ be a compressed sequence in $\mathbb{Z} \backslash\{0\}$ with $a_{k}<0$, and let $m \in \mathbb{Z}$ be any number expressible in the form $\sum_{t=1}^{k} \alpha_{t} a_{t}$ with each $\alpha_{t} \in \mathbb{N}$. Let $M$ be a matrix (with finitely many nonzero entries in each row) such that

(i) the compressed form of each row is $\left\langle a_{1}, a_{2}, \ldots, a_{k}\right\rangle$,

(ii) the sum of each row is $m$, and

(iii) any row with compressed form $\left\langle a_{1}, a_{2}, \ldots, a_{k}\right\rangle$ and row sum $m$ occurs in $M$. Then $M$ is not strongly centrally image partition regular, and if $m \leq 0, M$ is not centrally image partition regular.

Proof. If $M \vec{x} \in \mathbb{N}^{\omega}$ and either the entries of $M \vec{x}$ corresponding to distinct rows are distinct or $m \leq 0$, there must be infinitely many distinct entries in $\vec{x}$. This in turn forces some entries of $M \vec{x}$ to be negative.

Recall that we have defined the (binary) support of a positive integer $n$ by $n=\sum_{t \in \operatorname{supp}(n)} 2^{t}$. We introduce now some special notation needed for the proof of Theorem 3.14

Definition 3.12. Let $n \in \mathbb{N}$. The set $H$ is a block of $n$ if and only if $H$ is a maximal set of consecutive integers contained in $\operatorname{supp}(n)$. Also, $b(n)$ is the number of blocks of $n$.

Thus if, written in binary, $n=10011101011$, then the blocks of $n$ are $\{0,1\},\{3\}$, $\{5,6,7\}$, and $\{10\}$, and so $b(n)=4$.

Lemma 3.13. Let $B=\{n \in \mathbb{N}: b(n) \equiv 0(\bmod 2)\}$.

(a) For $n \in \mathbb{N}$, let $h(n) \in\{0,1\}$ be such that $h(n) \equiv b(n)(\bmod 2)$. Let $\widetilde{h}: \beta \mathbb{N} \rightarrow$ $\mathbb{Z}_{2}$ be the continuous extension of $h$. Then the restriction of $\widetilde{h}$ to $\bigcap_{n=1}^{\infty} c \ell\left(\mathbb{N} 2^{n}\right)$ is a homomorphism.

(b) If $q+q=q \in \beta \mathbb{N}$, then $B \in q$.

(c) If $p \in \beta \mathbb{N}$ and for each $n \in \mathbb{N}, \mathbb{N} 2^{n} \in p$, then $B \notin-p+p$.

Proof. (a) This is an immediate consequence of [7. Theorem 4.21].

(b) By [7 Lemma 6.6] $q \in \bigcap_{n=1}^{\infty} c \ell\left(\mathbb{N} 2^{n}\right)$; so this follows from (a).

(c) Let $D_{0}=\{x \in \mathbb{N}: \min (\operatorname{supp}(x))+1 \notin \operatorname{supp}(x)\}$ and let $D_{1}=\{x \in \mathbb{N}$ : $\min (\operatorname{supp}(x))+1 \in \operatorname{supp}(x)\}$.

Notice that if $x, y \in D_{0}$ and $\max (\operatorname{supp}(x))+1<\min (\operatorname{supp}(y))$, then $b(y-x)=$ $b(y)+b(x)-1$, while if $x, y \in D_{1}$ and $\max (\operatorname{supp}(x))+1<\min (\operatorname{supp}(y))$, then 
$b(y-x)=b(y)+b(x)+1$. Hence, in either case, if $b(x) \equiv b(y)(\bmod 2)$, then $y-x \notin B$.

Pick $t \in\{0,1\}$ such that $D_{t} \in p$. Also pick $i \in\{0,1\}$ such that $C \in p$, where $C=\{x \in \mathbb{N}: b(x) \equiv i(\bmod 2)\}$. Now $\left\{y-x: x, y \in C \cap D_{t}\right.$ and $\max (\operatorname{supp}(x))+1<\min (\operatorname{supp}(y))\} \in-p+p$ by [7. Theorem 4.15]. We have seen that this set does not meet $B$; so $B \notin-p+p$.

Theorem 3.14. Let $A$ be an $\omega \times \omega$ matrix with the property that $\vec{r} \in \mathbb{Q}^{\omega}$ occurs as a row of $A$ if and only if the nonzero entries of $\vec{r}$ are $1,-1,-1,1$, in that order. Then there is a subset $B$ of $\mathbb{N}$ that is a member of every idempotent in $\beta \mathbb{N}$ and there is no $\vec{x} \in \mathbb{N}^{\omega}$ for which all the entries of $A \vec{x}$ are in $B$.

Proof. Let $B=\{n \in \mathbb{N}: b(n) \equiv 0(\bmod 2)\}$ and suppose we have $\vec{x} \in \mathbb{N}^{\omega}$ with $A \vec{x} \in B^{\omega}$. Note that we cannot have more than three entries of $\vec{x}$ with the same value, since otherwise 0 would be an entry of $A \vec{x}$. Thus we may pick $q \in \mathbb{N}^{*} \cap\left\{x_{n}\right.$ : $n \in \omega\}$. Since $\left\{x_{n_{1}}-x_{n_{2}}-x_{n_{3}}+x_{n_{4}}: n_{1}<n_{2}<n_{3}<n_{4}\right\} \subseteq B$, we have that $B \in q+-q+-q+q$.

Let $p=-q+q$. Then $p \in \beta \mathbb{N}$ by [7, Exercise 4.3.5]. Also $-p=q+-q$ by 7. Lemma 13.1], so that $B \in-p+p$. It is easy to check that $\mathbb{N} 2^{n} \in p$ for each $n \in \mathbb{N}$ by picking $i \in\left\{0,1, \ldots, 2^{n}-1\right\}$ such that $\mathbb{N} 2^{n}+i \in q$. Thus we have a contradiction to Lemma 3.13 (c).

Corollary 3.15. Let $M$ be a matrix (with finitely many nonzero entries in each row) such that

(i) the compressed form of each row is $\langle 1,-1,1\rangle$,

(ii) the sum of each row is 0 , and

(iii) any row with compressed form $\langle 1,-1,1\rangle$ and row sum 0 occurs in $M$.

Then $M$ is not centrally image partition regular.

Proof. The matrix $M$ includes all the rows of the matrix $A$ of Theorem 3.14 .

Let $\mathcal{F}$ denote the set of finite image partition regular matrices over $\mathbb{Q}$. For $F \in \mathcal{F}$, let $C(F)$ be defined as in Definition 2.4 We know, from Theorem 1.2(b) and Lemma 2.5(b), that $\bigcap_{F \in \mathcal{F}} C(F)$ contains the smallest closed subsemigroup of $\beta \mathbb{N}$ containing the minimal idempotents. We now see that $\bigcap_{F \in \mathcal{F}} C(F)$ contains elements which do not belong to this semigroup.

Corollary 3.16. The set $\bigcap_{F \in \mathcal{F}} C(F)$ contains elements which do not belong to the smallest closed subsemigroup of $\beta \mathbb{N}$ containing the idempotents.

Proof. Let $A$ be the matrix defined in Theorem 3.14 Let $B=\{n \in \mathbb{N}: b(n) \equiv$ $0(\bmod 2)\}$. By Theorem 3.13 a) and the fact from 7, Lemma 6.6] that each idempotent is in $\bigcap_{n=1}^{\infty} c \ell_{\beta \mathbb{N}}\left(\mathbb{N} 2^{n}\right)$, we have that $c l_{\beta \mathbb{N}}(B)$ contains the smallest closed subsemigroup of $\beta \mathbb{N}$ that contains the idempotents. By Theorem 3.14, $C(A) \cap$ $c \ell_{\beta \mathbb{N}}(B)=\emptyset$. However, by Corollary 2.6. $C(A) \cap \bigcap_{F \in \mathcal{F}} C(F) \neq \emptyset$.

On the other hand, we see that some matrices with all row sums equal to 0 are strongly centrally image partition regular.

Theorem 3.17. Let $A$ be an $\omega \times \omega$ matrix whose entries are all in $\{-1,0,1\}$. Assume that

(i) the number of nonzero entries in each row is positive but finite,

(ii) the last nonzero entry in each row is 1 , and 
(iii) the nonzero entries in each row alternate in sign.

Then, $A$ is strongly centrally image partition regular. In fact, if $P$ is a member of any idempotent in $\beta \mathbb{N}$, then there is an increasing sequence $\vec{x} \in \mathbb{N}^{\omega}$ such that all the entries of $A \vec{x}$ are in $P$ and entries corresponding to distinct rows are distinct.

Proof. We can choose a sequence $\left\langle v_{n}\right\rangle_{n=0}^{\infty}$ such that $F S\left(\left\langle v_{n}\right\rangle_{n=0}^{\infty}\right) \subseteq P$ by [7, Theorem 5.8]. By taking sums of terms, we may suppose that $v_{n+1}>\sum_{i=0}^{n} v_{i}$ for every $n \in \omega$, and hence that $\sum_{i \in F_{1}} v_{i} \neq \sum_{i \in F_{2}} v_{i}$ if $F_{1}$ and $F_{2}$ are distinct members of $\mathcal{P}_{f}(\omega)$. For every $n \in \omega$, let $x_{n}=\sum_{i=0}^{n} v_{i}$.

Suppose that $\vec{r} \in\{-1,0,1\}^{\omega} \backslash \overrightarrow{0}$ has a finite number of nonzero entries and that its nonzero entries alternate in sign. Let $r_{n}$ be the last nonzero entry of $\vec{r}$. It is easy to see, by induction on the number of nonzero entries in $\vec{r}$, that there exists $F \in \mathcal{P}_{f}(\omega)$ such that $\max (F)=n$, and $\vec{r} \cdot \vec{x}=\sum_{i \in F} v_{i}$ if $r_{n}=1$, while $\vec{r} \cdot \vec{x}=$ $-\sum_{i \in F} v_{i}$ if $r_{n}=-1$. It follows that the entries of $A \vec{x}$ are in $P$. It is simple to verify that entries corresponding to distinct rows are distinct.

We do not know whether there is any matrix with all rows having a fixed compressed form and zero sums which is centrally image partition regular. We see however, that if such exists, it is also strongly centrally image partition regular.

Theorem 3.18. Let $k \in \mathbb{N}$, let $\left\langle a_{1}, a_{2}, \ldots, a_{k}\right\rangle$ be a compressed sequence in $\mathbb{Z} \backslash\{0\}$ with $a_{k}>0$, and assume that 0 is expressible in the form $\sum_{t=1}^{k} \alpha_{t} a_{t}$ with each $\alpha_{t} \in \mathbb{N}$. Let $M$ be a matrix (with finitely many nonzero entries in each row) such that

(i) the compressed form of each row is $\left\langle a_{1}, a_{2}, \ldots, a_{k}\right\rangle$,

(ii) the sum of each row is 0 , and

(iii) any row with compressed form $\left\langle a_{1}, a_{2}, \ldots, a_{k}\right\rangle$ and row sum 0 occurs in $M$. If $M$ is centrally image partition regular, then $M$ is strongly centrally image partition regular.

Proof. Let $C$ be central in $\mathbb{N}$ and pick $\vec{x} \in \mathbb{N}^{\omega}$ such that $M \vec{x} \in C^{\omega}$. We claim first that no value of $\vec{x}$ repeats infinitely often. Suppose instead that we have $d$ such that $\left|\left\{n \in \mathbb{N}: x_{n}=d\right\}\right|=\omega$. Pick $b_{1}, b_{2}, \ldots, b_{s}$ such that $\sum_{t=1}^{s} b_{t}=0$ and $c\left(\left\langle b_{1}, b_{2}, \ldots, b_{s}\right\rangle\right)=\vec{a}$. Pick $t_{1}<t_{2}<\ldots<t_{s}$ such that $x_{t_{1}}=x_{t_{2}}=\ldots=x_{t_{s}}=d$. Pick a row $i$ of $M$ that has $m_{i, t_{j}}=b_{j}$ for each $j \in\{1,2, \ldots, s\}$ and all other entries equal to 0 . Then $\sum_{j=0}^{\infty} m_{i, j} x_{j}=\sum_{j=1}^{s} b_{j} d=0 \notin C$, a contradiction.

Let $m=\max \left\{\left|a_{j}\right|: j \in\{1,2, \ldots, k\}\right\}$. Since no value of $\vec{x}$ repeats infinitely often, choose an infinite $B \subseteq \omega$ such that for each $n \in B$ with $n>\min B$,

$$
x_{n}>2 m \cdot \sum\left\{x_{t}: t \in B \text { and } t<n\right\} .
$$

Let $D$ be the matrix consisting of those columns of $M$ corresponding to members of $B$. Then let $A$ be the matrix consisting of those rows of $D$ that sum to 0 . Let $\left\langle z_{n}\right\rangle_{n=0}^{\infty}$ enumerate in order $\left\{x_{n}: n \in B\right\}$. Then $A$ has all of the rows of $M$, $\vec{y}=A \vec{z} \in C^{\omega}$ and, by Lemma 3.5 if $i$ and $j$ are distinct rows of $A$, then $y_{i} \neq y_{j}$.

Piecewise syndetic subsets of $\mathbb{N}$ are characterized [7] Theorem 4.40] as those sets whose closure meets $K(\beta \mathbb{N})$. In particular, any central set is piecewise syndetic. Further, piecewise syndetic sets are guaranteed to contain substantial combinatorial structures. For example [7, Theorem 14.1], any piecewise syndetic subset of $\mathbb{N}$ contains arbitrarily long arithmetic progressions. 
We note that the matrix $A$ in the next theorem need not be centrally image partition regular. Indeed, if each $a_{k} \in \mathbb{N}, k>1$, and every row with compressed form $\left\langle a_{1}, a_{2}, \ldots, a_{k}\right\rangle$ occurs in $A$, then as a consequence of [2] Theorem 3.14], one has that any idempotent $p \in \beta \mathbb{N}$ has a member $P$ such that no $\vec{x} \in \mathbb{N}^{\omega}$ has $A \vec{x} \in P^{\omega}$.

Theorem 3.19. Let $k \in \mathbb{N}$ and let $\left\langle a_{1}, a_{2}, \ldots, a_{k}\right\rangle$ be a compressed sequence in $\mathbb{Z} \backslash\{0\}$ with $a_{k}>0$. Let $A$ be an $\omega \times \omega$ matrix with entries in $\mathbb{Z}$, such that each row has a finite number of nonzero entries and has $\left\langle a_{1}, a_{2}, \ldots, a_{k}\right\rangle$ as its compressed form. If $C$ is a piecewise syndetic subset of $\mathbb{N}$, then there exist $c \in \mathbb{N}$ and an increasing sequence $\vec{x} \in \mathbb{N}^{\omega}$ such that all the entries of $A \vec{x}$ are in $-c+C$ and entries corresponding to distinct rows are distinct.

Proof. Pick by [7 Theorem 2.8] a minimal left ideal $L$ of $\beta \mathbb{N}$ such that $\bar{C} \cap L \neq \emptyset$, and pick $s \in \bar{C} \cap L$. Pick by [7, Corollary 2.6] an idempotent $r \in L$. Let $p=\frac{1}{a_{k}} \cdot r$ and let $q=a_{1} \cdot p+a_{2} \cdot p+\ldots+a_{k} \cdot p$. Then, as in the proof of Theorem 3.7, $p$ is an idempotent and $q \in L$. Thus $L=l+q$ by [7] Lemma 1.52]; so $s=t+q$ for some $t \in L$. Since $C \in s$, there is some $c \in \mathbb{N}$ such that $-c+C \in q$. The conclusion now follows by Corollary [3.6.

\section{REFERENCES}

1. W. Deuber, Partitionen und lineare Gleichungssysteme, Math. Zeit. 133 (1973), 109-123. MR 48:3753

2. W. Deuber, N. Hindman, I. Leader, and H. Lefmann, Infinite partition regular matrices, Combinatorica 15 (1995), 333-355. MR 96i:05173

3. H. Furstenberg, Recurrence in ergodic theory and combinatorial number theory, Princeton University Press, Princeton, 1981. MR 82j:28010

4. R. Graham, B. Rothschild, and J. Spencer, Ramsey Theory, Wiley, New York, 1990. MR 90m:05003

5. N. Hindman and I. Leader, Image partition regularity of matrices, Comb. Prob. and Comp. 2 (1993), 437-463. MR 95j:05167

6. N. Hindman, I. Leader, and D. Strauss, Image partition regular matrices - bounded solutions and preservation of largeness, Discrete Math. 242 (2002), 115-144. MR 2002j:05146

7. N. Hindman and D. Strauss, Algebra in the Stone-Cech compactification - theory and applications, W. de Gruyter \& Co., Berlin, 1998. MR 99j:54001

8. K. Milliken, Ramsey's Theorem with sums or unions, J. Combinatorial Theory (Series A) 18 (1975), 276-290. MR 51:10106

9. R. Rado, Studien zur Kombinatorik, Math. Zeit. 36 (1933), 242-280.

10. I. Schur, Über die Kongruenz $x^{m}+y^{m}=z^{m}(\bmod p)$, Jahresbericht der Deutschen Math.Verein. 25 (1916), 114-117.

11. A. Taylor, A canonical partition relation for finite subsets of $\omega$, J. Combinatorial Theory (Series A) 21 (1976), 137-146. MR 54:12530

12. B. van der Waerden, Beweis einer Baudetschen Vermutung, Nieuw Arch. Wiskunde 19 (1927), 212-216.

Department of Mathematics, Howard University, Washington, DC 20059

E-mail address: nhindman@aol.com

URL: http://members.aol.com/nhindman/

Department of Pure Mathematics and Mathematical Statistics, University of CamBRidge, Cambridge CB2 1SB, United Kingdom

E-mail address: I.Leader@dpmms.cam.ac.uk

Department of Pure Mathematics, University of Hull, Hull HU6 7RX, United KingDOM

E-mail address: d.strauss@maths.hull.ac.uk 